PALEO

Revue d'archéologie préhistorique

19 | 2007

Spécial table ronde ( 1 ère partie) : Le Gravettien : entités régionales d'une paléoculture européenne, Les Eyzies, juillet 2004

\title{
Variabilité du Gravettien de Kostienki (Bassin moyen du Don) et des territoires associés
}

Gravettian variability in Kostienki (Don middle basin) and in linked territories

\section{Andey A. Sinitsyn}

\section{OpenEdition}

\section{Journals}

Édition électronique

URL : https://journals.openedition.org/paleo/599

DOI : 10.4000/paleo.599

ISSN : 2101-0420

Éditeur

SAMRA

Édition imprimée

Date de publication : 30 décembre 2007

Pagination : 181-201

ISSN : $1145-3370$

Référence électronique

Andey A. Sinitsyn, « Variabilité du Gravettien de Kostienki (Bassin moyen du Don) et des territoires associés », PALEO [En ligne], 19 | 2007, mis en ligne le 23 avril 2009, consulté le 24 juin 2021. URL: http://journals.openedition.org/paleo/599; DOI : https://doi.org/10.4000/paleo.599

\section{(c) (i) $\odot$}

PALEO est mis à disposition selon les termes de la licence Creative Commons Attribution - Pas d'Utilisation Commerciale - Pas de Modification 4.0 International. 


\title{
VARIABILITÉ DU GRAVETTIEN DE KOSTIENKI (Bassin moyen du Don) ET DES TERRITOIRES ASSOCIÉS
}

\author{
Andey A.SINITSYN ${ }^{(1)}$
}

\begin{abstract}
Résumé : Dans la région de Kostienki-Borschevo, on observe l'expression, à ce jour, la plus orientale du modèle européen de l'évolution du Paléolithique supérieur. Elle est différente à la fois du modèle Sibérien et du modèle de l'Asie centrale. Comme ailleurs en Europe, le Gravettien apparaît à Kostienki vers 28 ka (Kostienki 8 /II/). Par la suite, entre 24-20 ka, les techno-complexes gravettiens sont représentés au moins par quatre faciès dont deux, ceux de Kostienki 21 /III/ et Kostienki 4 /II/, ressemblent au Gravettien occidental et deux autres, Kostienki-Avdeevo et Kostienki 11 /II/, sont des faciès propres à l'Europe de l'Est, sans analogie à l'Ouest.
\end{abstract}

Mots-clés : Europe orientale, Paléolithique, Kostienki-Borschevo, Gravettien.

Abstract: Until now, the Kostienki-Borshchevo area appears to be the most eastern manifestation of the general european model of the Upper Palaeolithic evolution, distinct from Siberian and Central Asian models. As well as in various parts of the European continent Gravettian occurs at Kostienki suddenly, around $28 \mathrm{ka}$ (Kostenki 8 /II/). At the following stage at 24-20 ka, the gravettian technocomplex are reprensented, at least by four versions, two of them - Kostienki $21 / \mathrm{III} / \mathrm{type}$, and Kostienki $4 / \mathrm{II} / \mathrm{type}-$ have western european typological configuration; and two others - Kostenki-Avdeevo type, and Kostenki $11 / \mathrm{ll} /$ type - appear to be specific to the east european facies, without analogies in the West.

Key-words: EastemEurope, Palaeolithic, Kostenki-Borshchevo, Gravettian.

La région de Kostienki comporte 26 sites paléolithiques dont la moitié contient plusieurs couches archéologiques. Cet ensemble représente actuellement plus de 50 niveaux d'habitat en position stratigraphique. Une telle concentration est exceptionnelle, mais elle n'est pas unique, des densités comparables sont connues par exemple dans la région des Eyzies en Dordogne ou dans la région de Pavlov en Moravie. Ce qui est propre à Kostienki est un modèle particulier d'évolution culturelle, distinct de celui d'autres régions du monde paléolithique. En dépit d'une faible superficie, de l'ordre de 20 kilomètres carrés, par rapport aux vastes zones de steppe du Dniepr moyen ou de l'Oural, la région de Kostienki-Borschevo constitue une véritable province au sein du monde paléolithique de l'Europe de l'Est.
La province de Kostienki fait partie du monde paléolithique européen, clairement distincte du monde Sibérien et du monde d'Asie centrale. C'est actuellement l'avancée la plus orientale de la manifestation du modèle général européen de l'évolution, dans laquelle sont présents des caractères propres à l'Europe en association avec des particularités locales spécifiques.

\section{1 - KOSTIENKI (fig.1) ${ }^{2}$}

Vers 1950-1960, les ensembles culturels des sites de Kostienki-Borschevo étaient répartis par A.N. Rogatchev (1957) en trois groupes chronologiques d'après leurs positions géologiques. Deux groupes anciens étaient liés à

(1) Institut d'Histoire de la Culture Matérielle, Académie des Sciences de Russie. Dvortsovaia nab., 18. 191186 SaintPétersbourg-a.sinitsyn@as6238.spb.edu

(2) Le numéro des sites est désigné par les chiffres arabes, le numéro du niveau archéologique par les chiffres romains entre parenthèses. 


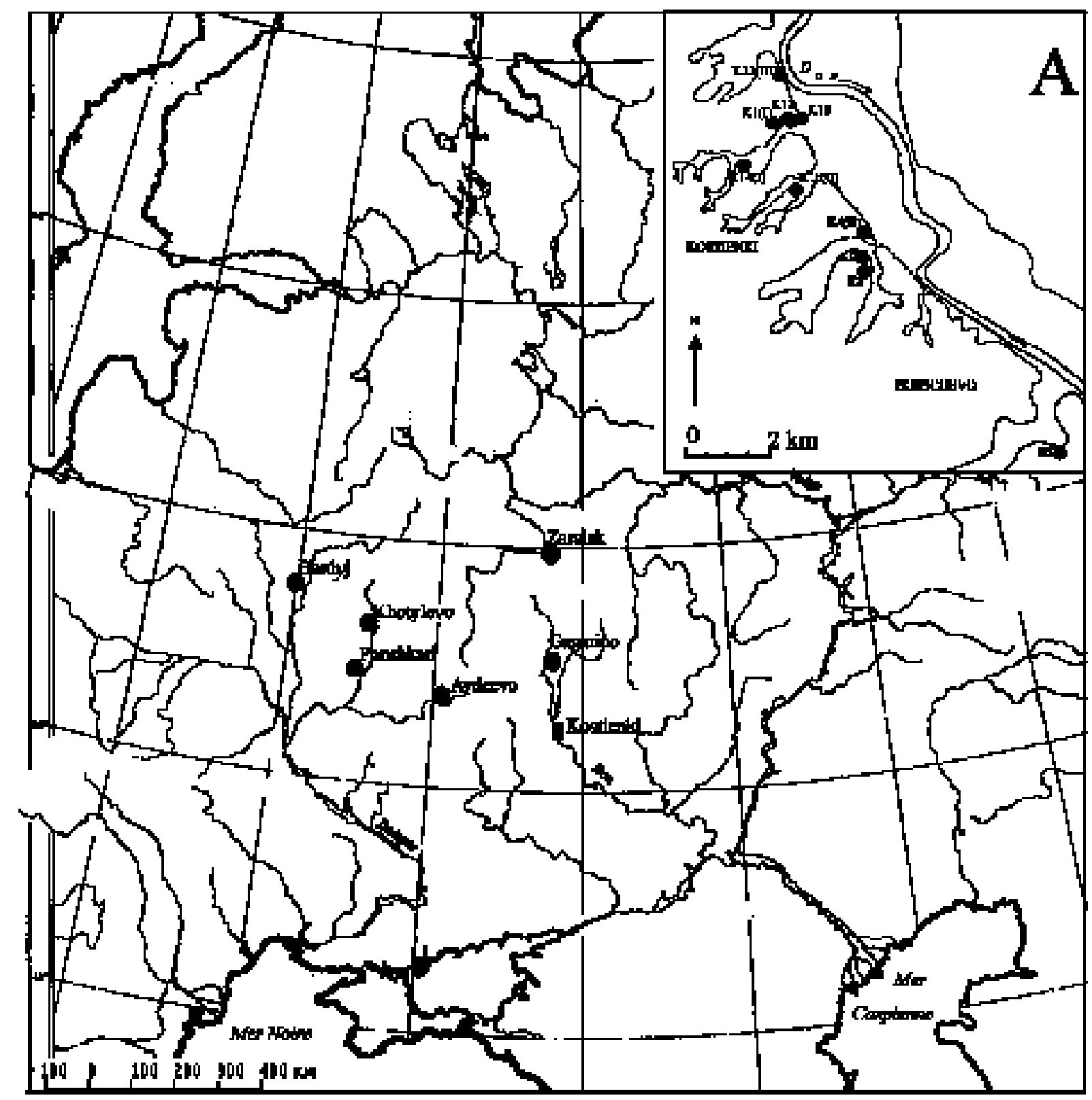

Figure 1 - Localisation des sites mentionnés dans le texte en Europe de l'Est et des sites gravettiens dans la région de Kostienki-Borschevo.

Figure 1 - Location of the sites mentioned in the texte in Earstern Europe and gravettian sites from Kostienki-Borschevo area.

deux horizons d'humus séparés par des cendres volcaniques, le troisième, plus récent, est associé à des sédiments loessiques argileux. Dans les années 1980, sur la base de séries des datations absolues, le cadre chronologique de ces industries a été défini comme suit :

I (ancien) : 36-32 ka ;

II (moyen) : 32-28 ka;

III (récent) : 27-20 ka (Praslov et Soulerjytsky 1997 ; Sinitsyn et al. 1997 ; Sinitsyn 1999). Le groupe récent du modèle chronologique de Kostienki correspond au stade moyen du Paléolitique supérieur du schéma européen.
Jusqu'à une période récente, la structure du groupe ancien était définie par la coexistence des faciès Streletskien et Spytsynien (Praslov et Rogatchev 1982 ; Boriskovski 1984). La découverte d'un niveau aurignacien dans de la cendre volcanique (Sinitsyn 2003a) et l'identification d'une nouvelle tradition culturelle dans la couche inférieure (IVb) de Kostienki 14 (Markina gora) ont conduit à diviser cet ensemble en deux sous-groupes : le plus ancien ou Paléolithique supérieur initial (42-36 ka) où coexistent le faciès Spytsynien et l'industrie de la couche inférieure de Markina gora; puis le Paléolithique supérieur 
ancien (36-32 ka), avec la coexistence de Streletskien et d'Aurigacien (Sinitsyn 2003b).

La composition du groupe moyen (32-28 ka) avait été définie par la coexistence d'Aurignacien, de Streletskien, de Gorodcovien (entité propre à l'Europe de l'Est) et de Gravettien. Sur la base de datations absolues, ce groupe peut être maintenant divisé en deux sous-groupes chronologiques ; le plus ancien s'inscrivant dans la continuité des premières unités culturelles (Aurignacien et Streletskien) le plus récent, correspondant à l'apparition de Gravettien et de Gorodcovien.

La structure du groupe récent de la séquence locale (24$20 \mathrm{ka}$ ) est définie par la coexistence de quatre faciès de Gravettien, en association avec des assemblages originaux dont la dénomination fait encore débat. II s'agit des sites de Kostienki 2, 3 et 19 qui sont contemporains mais pas gravettiens.

\section{2- GRAVETTIEN ANCIEN. KOSTIENKI 8 (Telmanskaia) (II)}

L'assemblage de la couche II de Kostienki 8 est la seule industrie attribuée au Gravettien dans le groupe moyen du schéma chronologique de Kostienki (phase terminale du Paléolithique supérieur ancien du schéma européen). La date ${ }^{14} \mathrm{C}$ de $27700 \pm 750$ (GrN-10509) est considérée comme la limite supérieure de l'âge des ensembles de ce groupe, associés aux niveaux humiques supérieurs (32-28 ka).

C'est l'industrie de la couche II de Telmanskaya qui a permis de décrire un Gravettien de type méditerranéen (Efimenko 1953, 1956 et 1960) qui a de fortes analogies avec celui de la grotte Paglicci. Cette ressemblance est maintenant confirmée par un âge similaire (couche 23A : $28100 \pm 400$; 22F : $28300 \pm$ 400, Palma di Cesnola 1996). Le niveau d'occupation de la couche II de Telmanskaya est caractérisé par les restes de cinq structures d'habitat légères avec un foyer central (Litovtchenko 1966) comparables à celle qui a été décrite en Dordogne à Corbiac (Bordes 1968).

L'assemblage lithique compte près de 23000 objets. La technologie est caractérisée par une économie maximale de la matière première. Au vu de la taille très petite des nucléus, on peut penser qu'ils ont été utilisés jusqu'à la fin. La technique laminaire est largement majoritaire. L'utilisation des burins comme nucléus pour la production des supports semble particulièrement importante ici.

Plus de 2000 outils retouchés constituent $9 \%$ du nombre total des objets taillés. La composition typologique de l'industrie lithique est caractérisée par la prépondérance des burins (24 \%) sur des grattoirs (2,5\%) ; par la forte domination des lamelles à dos et pointes sur lamelles à bord abattu (43\%). Avec des pointes largement répandues, il y a une série de formes spécifiques, des bipointes variées, des trapèzes, des segments. Cette grande variété typologique de pointes, en association avec un haut degré de standardisation, témoigne du caractère évolué de l'industrie ou du moins, d'une relative importance de la composante microlithique. Les outils du groupe peu abondant des grattoirs, au contraire, se distinguent par l'absence de standardisation (fig. 2). Les denticulés et encoches, perçoirs et racloirs soulignent la composition particulière de l'industrie (Rogatchev
1957 ; Tchelidze 1968 ; Litovtchenko 1969).

L'industrie osseuse comprend des types largement répandus comme des bâtons en ivoire et en os, des alênes, des lissoirs. La parure est représentée par des perles longues en os d'oiseaux ou de petits mammifères, décorés d'incisions parallèles.

Le problème de l'appartenance culturelle de l'assemblage de la couche II de Kostienki 8 reste ouvert en raison de l'absence totale en Europe de l'Est de sites similaires contemporains de la période 27-28 ka.

\section{3 - GRAVETTIEN ÉVOLUÉ}

Le problème principal du Gravettien évolué (groupe moyen du schéma chronologique de Kostienki) est une grande variabilité avec de nombreux faciès aux fossiles directeurs spécifiques représentés par des industries provenant de sites sporadiques. A deux exceptions près, toutes ces variétés ne sont représentées qu'à Kostienki et n'ont pas d'équivalent en dehors de cette région.

\section{1 - Kostienki-Avdeevo (Kostienkovien)}

Le faciès Kostienki-Avdeevo est la seule entité culturelle présente à Kostienki dans quatre sites, mais on la trouve également hors des sites de Kostienki.

Tous les sites (fig. 1) sont localisés dans la ravine Prokrovsky et représentent probablement les restes d'une vaste zone d'habitation (Praslov et Rogatchev 1982).

La culture est caractérisée par un type spécifique d'organisation de l'espace, représenté par une grande habitation (36 x 14-15 m) avec de nombreux foyers en ligne sur l'axe longitudinal de l'habitation, entourée par des constructions semi-souterraines d'une profondeur d'un mètre et par des fosses-dépôts.

Les trois fossiles directeurs du Kostienkovien ont été décrits à l'issue des premières fouilles (Efimenko 1923). II s'agit de l'association de statuettes féminines réalistes, de pointes à cran aux dimensions spécifiques et des couteaux du type de Kostienki. Par la suite, le nombre des traits typologiques particuliers de cette unité culturelle locale d'Europe de l'Est a augmenté (Praslov et Rogachev 1982 ; Gvozdover 1998 ; Beliaeva 1977 et 1979 ; Giria et Bradley 1998), mais l'importance de l'association évoquée plus haut reste le critère diagnostique de cette culture (fig. 3).

En association avec les assemblages lithique et osseux nombreux et variés, les représentations féminines et animales et les objets de parure complexes, il faut mentionner des structures de la vie domestique (foyers, fosses ayant des fonctions différentes, etc.) un exemple de cette entité culturelle, Kostienki 18, a fourni des sépultures révélant un rite funéraire particulier (Praslov et Rogachev 1982).

Une série de 42 datations par le radiocarbone ont été obtenues pour Kostienki 1 (I). Elles se répartissent entre $18230 \pm 620$ (LE-3280) et $24100 \pm 500$ (GIN-2529) avec une moyenne proche de $22-23 \mathrm{ka}$; les dates pour Kostienki 14 (I) sont : $22500 \pm 1000$ (LE-5274) et 22780 $\pm 250(\mathrm{OxA}-4114)$ et pour Kostienki $18: 20600 \pm 140$ (GIN-8032) et $21020 \pm 180$ (OxA-7128). 


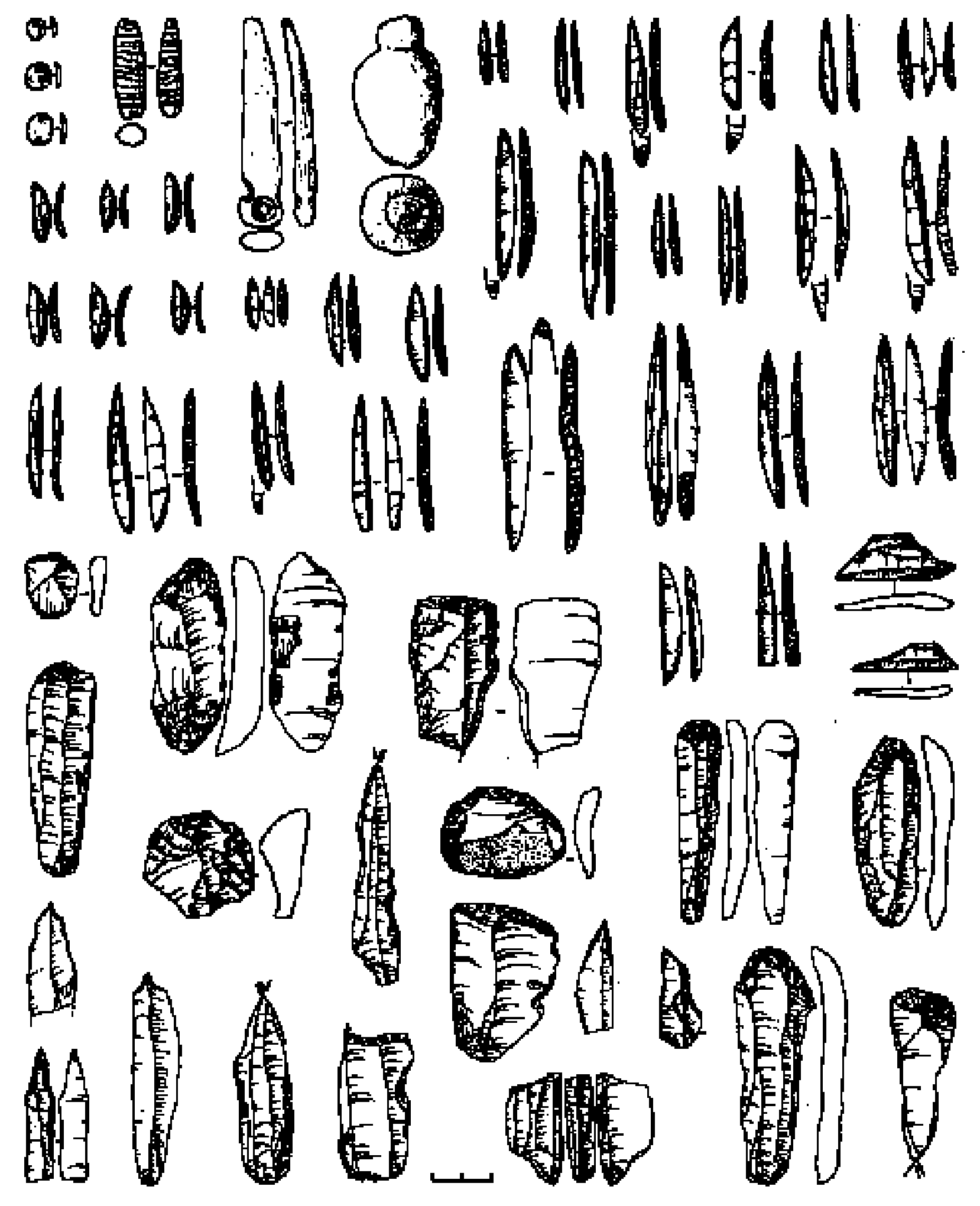

Figure 2 - Kostienki 8 (Telmanskaia) (II). L'assemblage lithique et osseux (d'après Rogatchev 1957; Litovtchenko 1968, 1969; Praslov et Rogachev 1982).

Figure 2 - Kostienki 8 (Telmanskaia) (II).Lithic and fauna 'assemblage (After Rogatchev 1957; Litovtchenko 1968, 1969; Praslov and Rogachev 1982). 


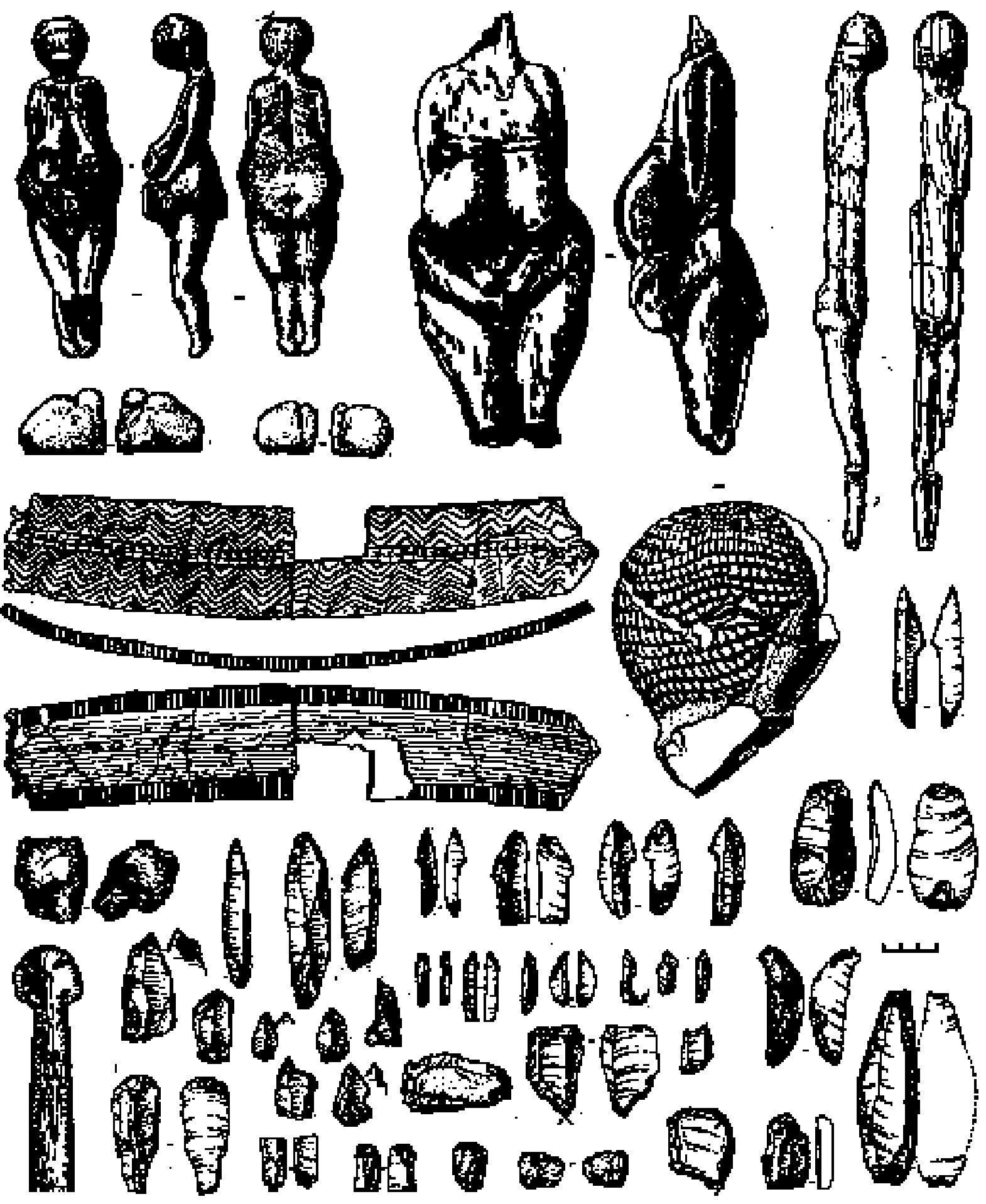

Figure 3 - Kostienki 1 (Poliakov) (I) (d'après Praslov et Rogachev 1982).

Figure 3 - Kostienki 1 (Poliakov) (I) (Afetr Praslov andt Rogachev 1982). 


\subsection{1 - Kostienkovien en dehors de Kostienki}

Les types diagnostiques de Kostienkovien ont également été trouvés dans les sites suivants:

- Avdeevo (fig. 4). Ce site est presque identique à Kostienki 1 (I) avec tous les marqueurs: organisation de l'habitat, assemblages lithique et osseux, art figuratif et éléments de parure (Abramova 1962, 1966, 1967 et 2005 ; Gvozdover 1961, 1995 et 1998). Les dates $22800 \pm 160$ (GIN-7724), $23140 \pm 430$ (GIN-7728a), $23400 \pm 700$ (GIN-7729) sont en conformité avec les données géologiques du site.

- Gagarino (fig. 5). Ce site se distingue des assemblages typiques de Kostienkovien par une composition des outillages différente, par la structure de l'espace d'habitation, par des détails stylistiques relatifs aux statuettes féminines, par des éléments d'assemblage osseux et par une technique de perforation biconique qui est inconnue à Kostienki 1 (I) et Avdeevo. Les traits les plus spécifiques des assemblage lithiques de Gagarino sont des proportions de pointes à cran différentes de celle d'Avdeevo et de Kostienki 1, des formes particulières de lamelles à cran et des couteaux de Kostienki (Zamiatnine 1934 ; Tarassov 1979). Une série de huit dates ont été obtenues, parmi lesquelles deux sont plus probables : 21 $600 \pm 140$ (GIN-7989) et $21800 \pm 300$ (GIN-1872).

- Khotylevo 2 (fig. 6). Comparées à Kostienki 1, Avdeevo et Gagarino, ce site a montré des différences remarquables. À côté de ressemblances bien marquées avec ces sites, en ce qui concerne la composition technologique et typologique des outillages, la particularité de Khotylevo est surtout marquée par le style des statuettes féminines, les objets de parure, un cas unique de décoration d'artéfacts lithiques sur le cortex et des outillages osseux très particuliers. La spécificité des outillages lithiques s'exprime par des proportions variables dans la composition typologique, ainsi que par la présence de types particuliers (Zaverniaev 1974, 1978, 1991et 2000 ; Gavrilov 2002 et 2004 ; Seleznev 1998). L'attribution de Khotylevo à l'entité culturelle de Kostienki-Avdeevo reste fortement problématique. Les dates $23660 \pm 270$ (LU-359), $24960 \pm 400$ (IGAN-73) ont été obtenues pour ce site.

- Zaraisk (fig. 7). Grâce à la découverte de figurines féminines en 2005 (Amirkhanov et Lev 2007), l'attribution de ce site au faciès Kostienki-Avdeevo ne fait pas de doute. II convient de noter une sculpture du bison d'un style totalement inconnu dans le Gravettien. Une grande dispersion des datations par radiocarbone et de nombreux détails microstratigraphiques posent le problème de la durée d'occupation du site (Troussov 1994, 1998 et 2002 ; Amirkhanov 1998 et 2000 ; Amirkhanov et Lev 2004 ; Lev et Amirkhanov 2002). Plus de 20 dates entre $15600 \pm 300$ (GIN-3700) et $23000 \pm 400$ (GIN8397a) ont été obtenues.

- Berdyj (fig. 8) Bien que dépourvus de statuettes, les fossiles directeurs du faciès Kostienkovien montrent des datations par radiocarbone de ce site $23430 \pm 180$ (LU-104). Elles semblent plus probables que l'âge de $15100 \pm 250$ (OxA-716) que proposent par ailleurs (Polikarpovitch 1968 ; Boudko 1964 et 1970 ; Boudko et Sorokina 1969 ; Kaletchetz 1984 ; Ksenzov 1988).

Bien que chacun de ces sites ait ses particularités, leur attribution à une tradition culturelle au sens large n'est pas mise en doute.
Les questions posées par les sites de Kostienki sont nombreuses et couvrent toutes les interrogations relatives au Paléolithique supérieur. Parmi les points essentiels d'un vaste débat, il faut citer la structure des industries, leur origine, leurs tendances évolutives, leurs relations avec les sites à pointes à cran d'Europe centrale et la position taxonomique du Kostienkien dans le cadre du Gravettien européen ou d'entitès intermédiaires du type Kostienki-Pavlov-Willendorf. L'hypothèse de la totale indépendance culturelle des sites, l'interprétation des différents sites comme habitats contemporains ou, au contraire, leur échelonnement dans le temps (Grigoriev 1993 ; Tarassov 1979 ; Anikovich 1998 ; Gavrilov 2004 ; Soffer 1987 ; 1993a et b ; Amirkhanov 1998 et 2000 ; Kozlowski 1969, 1970, 1986 et 1998 ; Otte et al. 1996).

\section{2 - FACIÈS DU TYPE DE KOSTIENKI 4 (Niveau II)}

Appelé également de type Alexandrov (l'autre nom de Kostienki 4 d'après le nom local du ravin où le site se tro uve), ce faciès représente l'autre variété de Gravettien dont les particularités sont les suivantes:

- un type unique de structures d'habitations longues ( 35 x 5.5 et $23 \times 5.5 \mathrm{~m}$ ), enterrées de $20-30 \mathrm{~cm}$ sous la paléosurface et des foyers disposés selon l'axe des constructions ;

- l'assemblage lithique caractérisé (fig. 9) par l'importance frappante des lames et pointes à bord abattu (41\%), des pièces esquillées de formes variées (17\% soit 1200 pièces). Aucun autre assemblage gravettien n'a livré ces objets dans de telles quantités. Quelques rares burins (2\%) et grattoirs (1\%) semblent être une autre caractéristique de cette industrie. Les pointes et lames à bord abattu sont marquées par une forte variabilité. Elles sont nettement différentes de celles de Telmanskaia et des assemblage de Kostienkovien et beaucoup plus proches des pointes du Gravettien occidental «classique».

L'industrie osseuse et les parures sont rares et représentées par des types largement répandus.

D'après l'avis de l'auteur des fouilles A.N. Rogatchev (1955; Praslov et Rogachev 1982) l'assemblage du niveau II de Kostienki 4 n' a pas d'analogie directe avec les sites contemporains de l'Europe de l'Est.

\section{3 - Variété du type de Kostienki 11 (Anosovka 2) (niveau II)}

L'association de structures d'habitations légères simples et circulaires, d'un diamètre proche de $6 \mathrm{~m}$, avec une industrie lithique originale et des objets d'art élaborés, parmi lesquels on note de petites figurines animales, est un des critères de base pour faire de cet assemblage une variété particulière du Gravettien.

L'outillage lithique de ce site comporte (Rogachev 1961 et 1962 ; Praslov et Rogachev 1982 ; Popov 1983 ; Popov et Pustovalov 2004 ; Popov et al. 2004 ; Bessoudnov 2005) (fig. 10) :

- un nombre important de lames tronquées, parfois obliques et concaves, formant la pointe d'un perçoir avec bord latéral retouché ;

- un indice de burins relativement bas (15\%) et de rares grattoirs (moins de $1 \%$ ) ;

- des outils sur lames pédonculées ; 

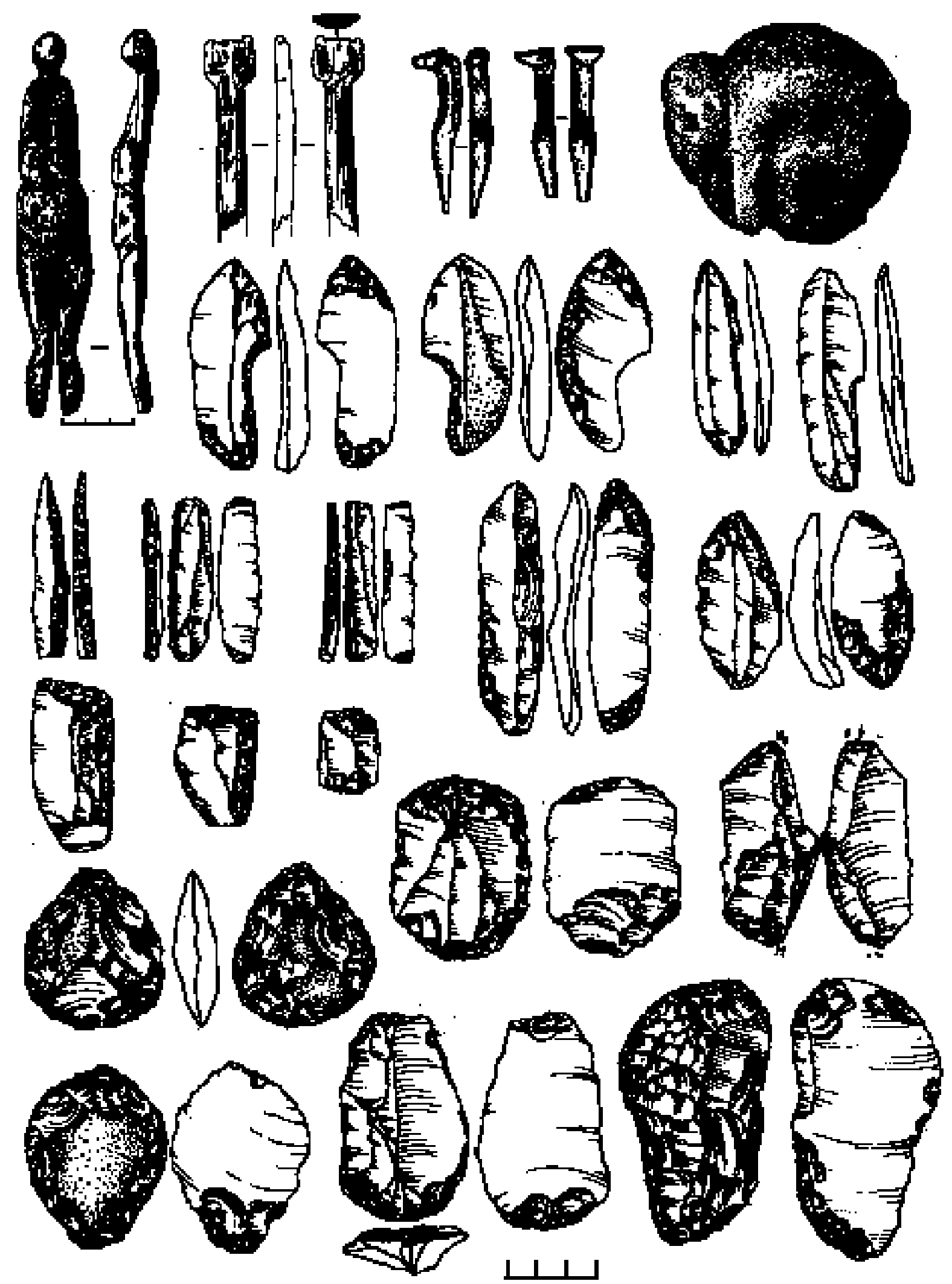

Figure 4 - Avdeevo (d'après Gvozdover, 1998; Abramova, 1962).

Figure 4 - Avdeevo (After Gvozdover 1998; Abramova 1962). 

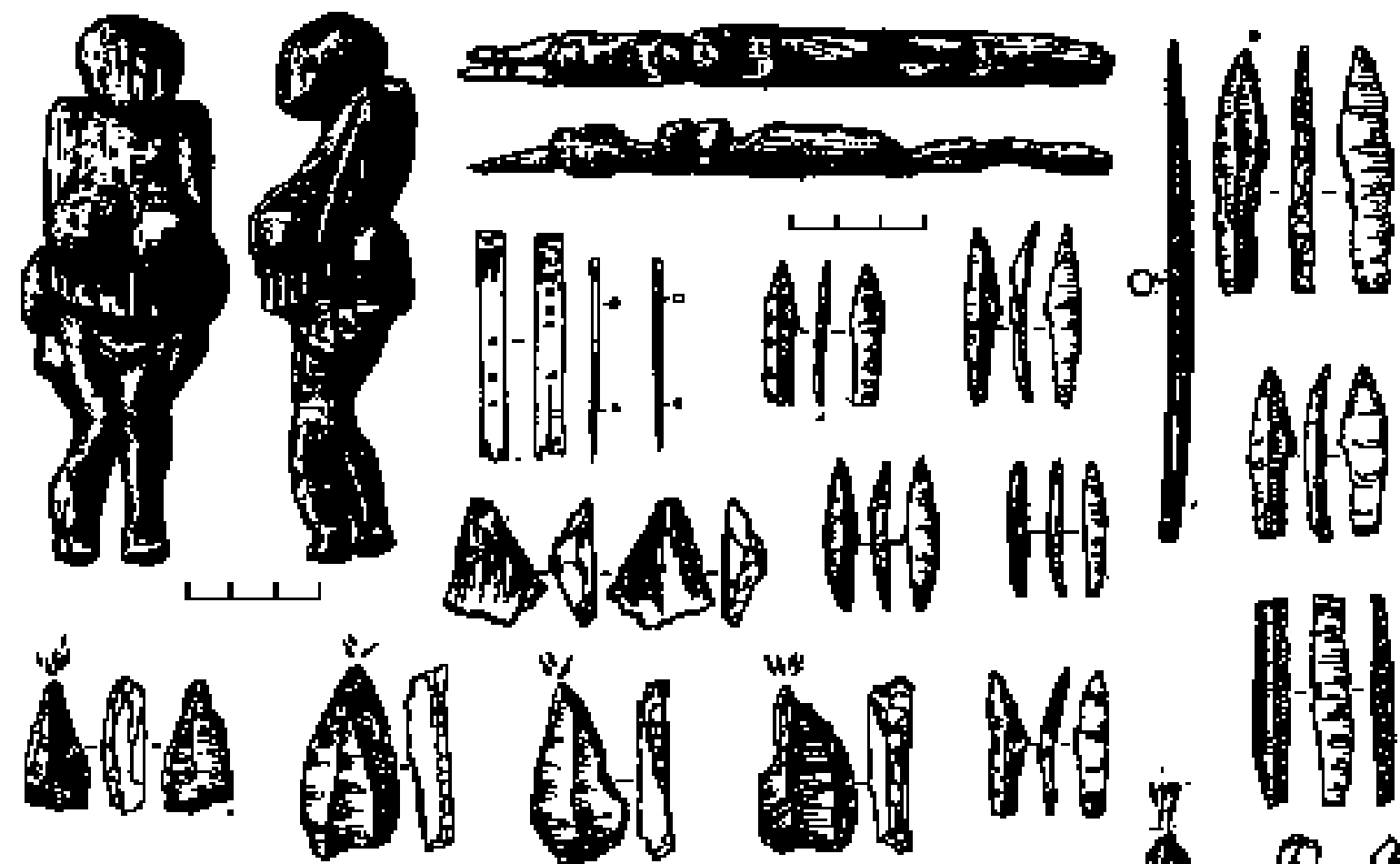

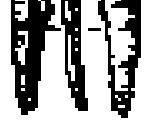

A A H H HO
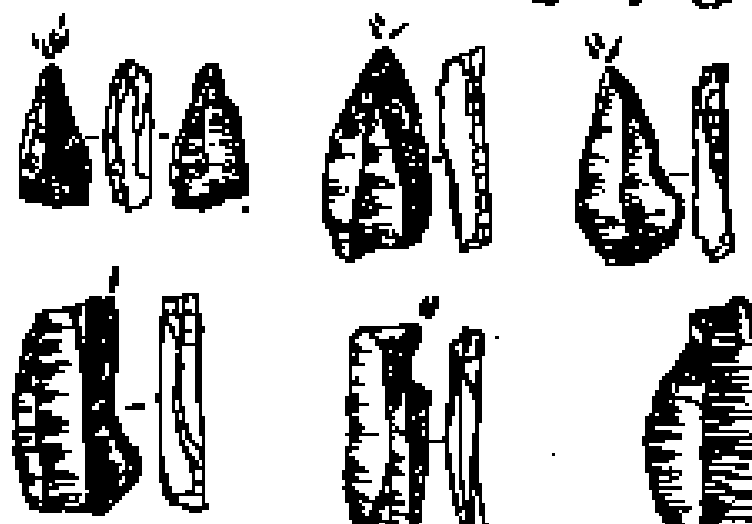

14t

)

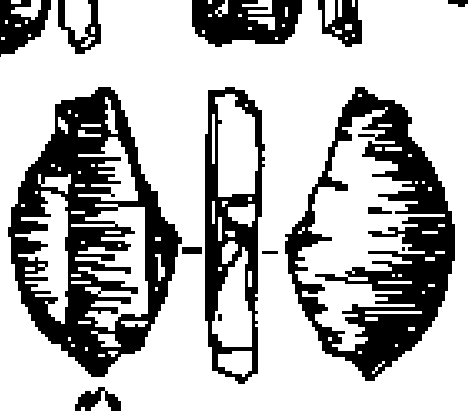

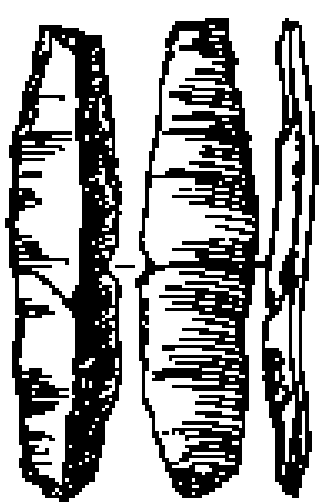

跑
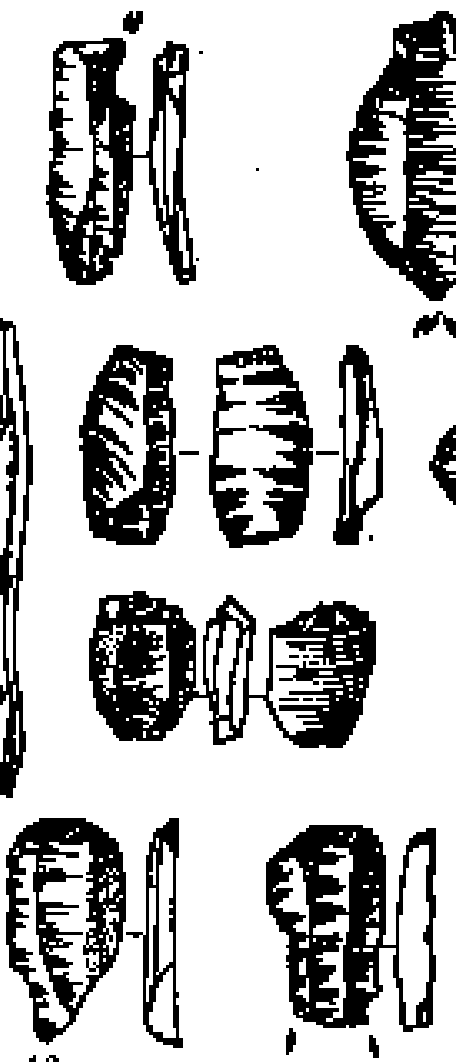
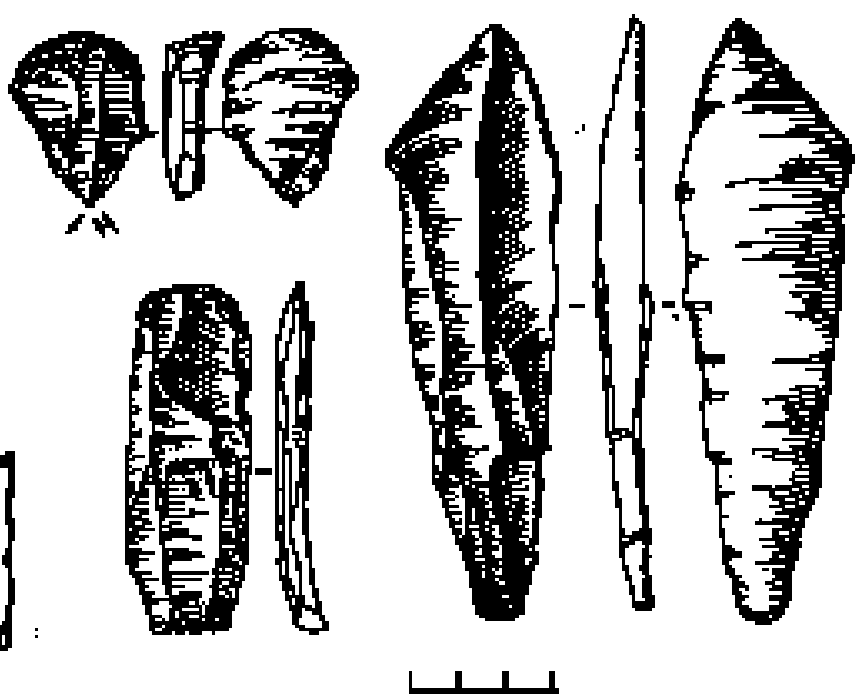

Figure 5 - Gagarino (d'après Tarassov 1979).

Figure 5 - Gagarino (After Tarassov 1979). 

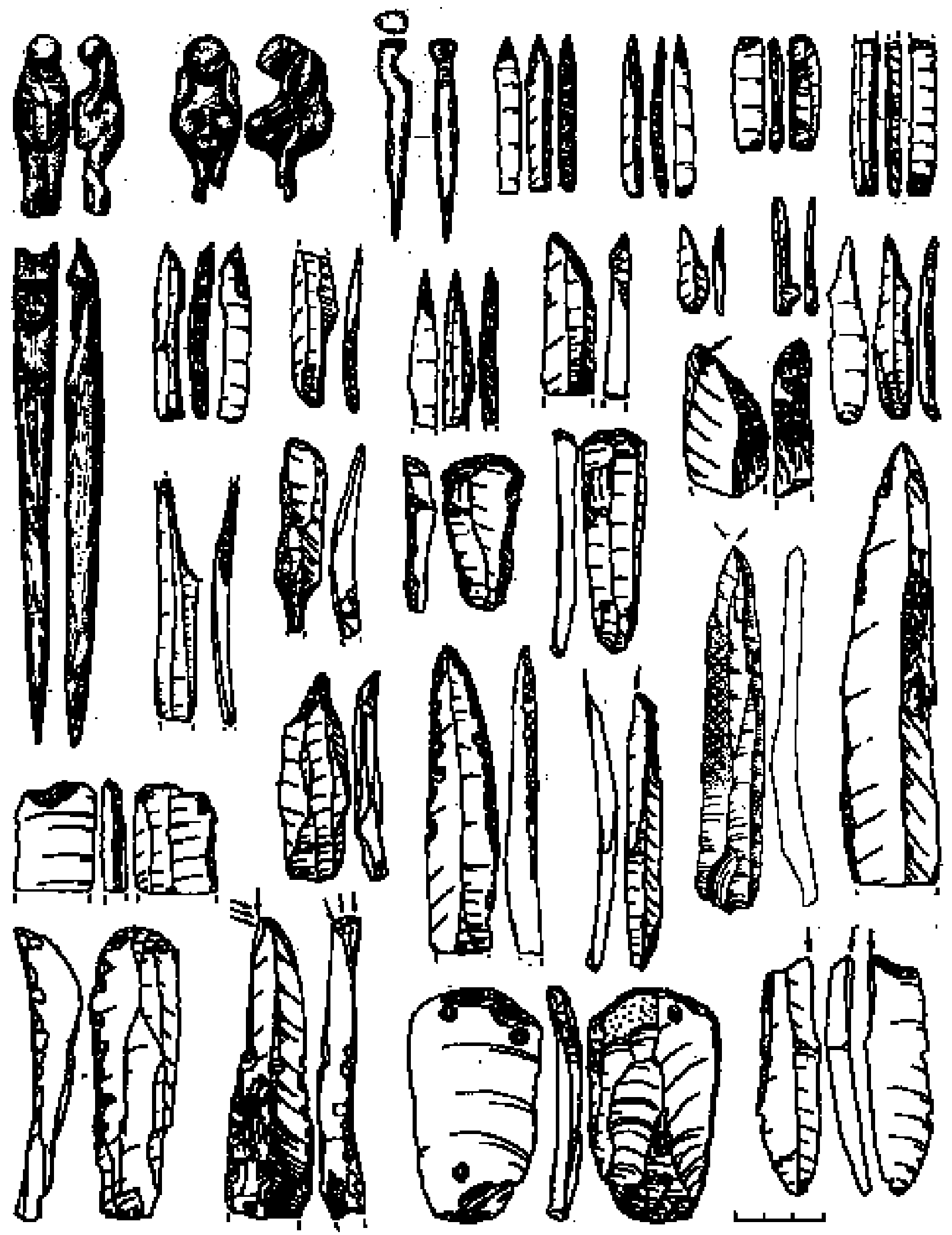

Figure 6 - Khotylevo 2 (d'après Zaverniaev 1974, 1978, 1991; Gavrilov 2004).

Figure 6 - Khotylevo 2 (After Zaverniaev 1974, 1978, 1991; Gavrilov 2004). 


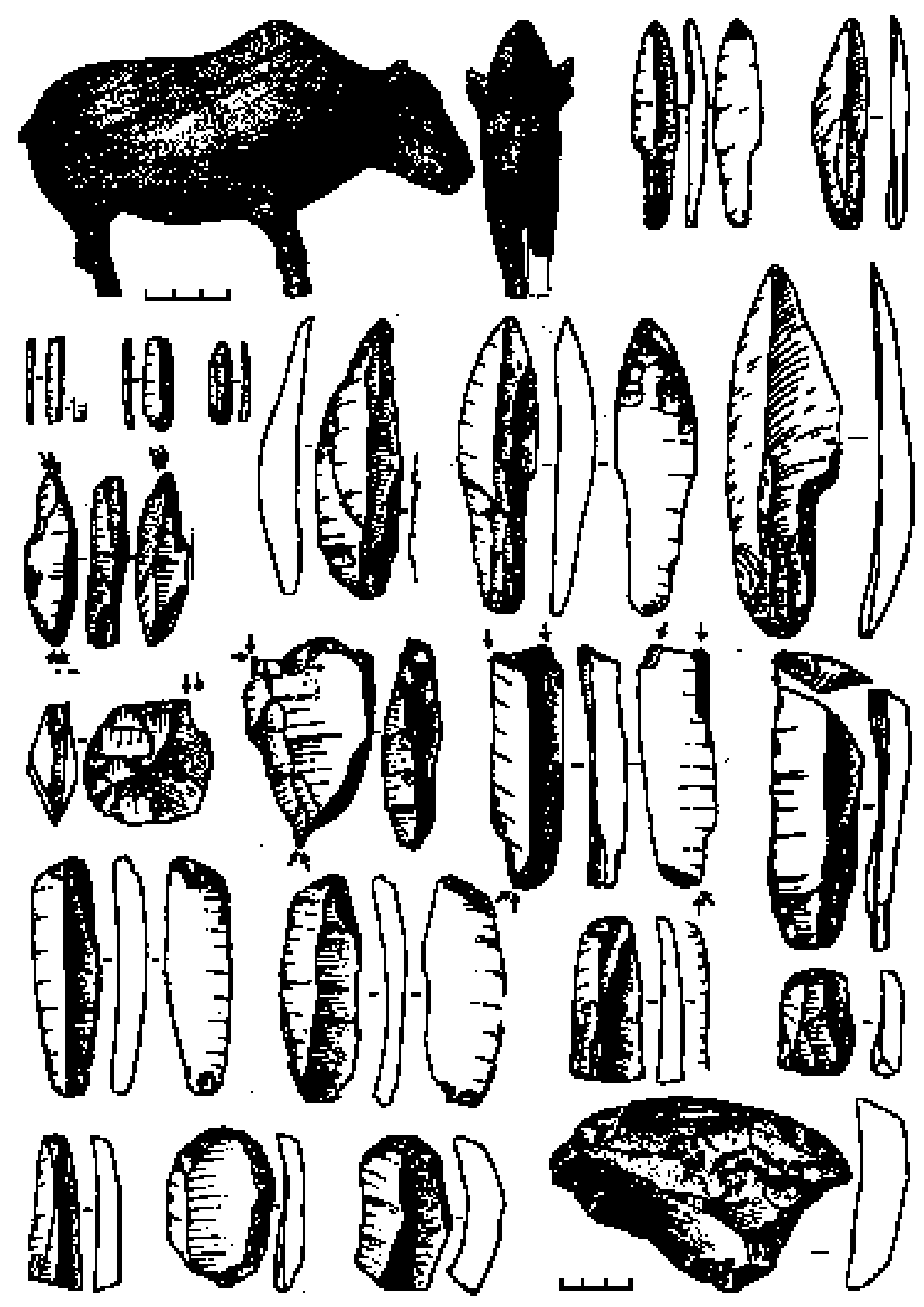

Figure 7 - Zaraisk (d'après Amirkhanov 2000; Amirkhanov et Lev 2004; Troussov 1998).

Figure 7 - Zaraisk (After Amirkhanov 2000; Amirkhanov and Lev 2004; Troussov 1998). 

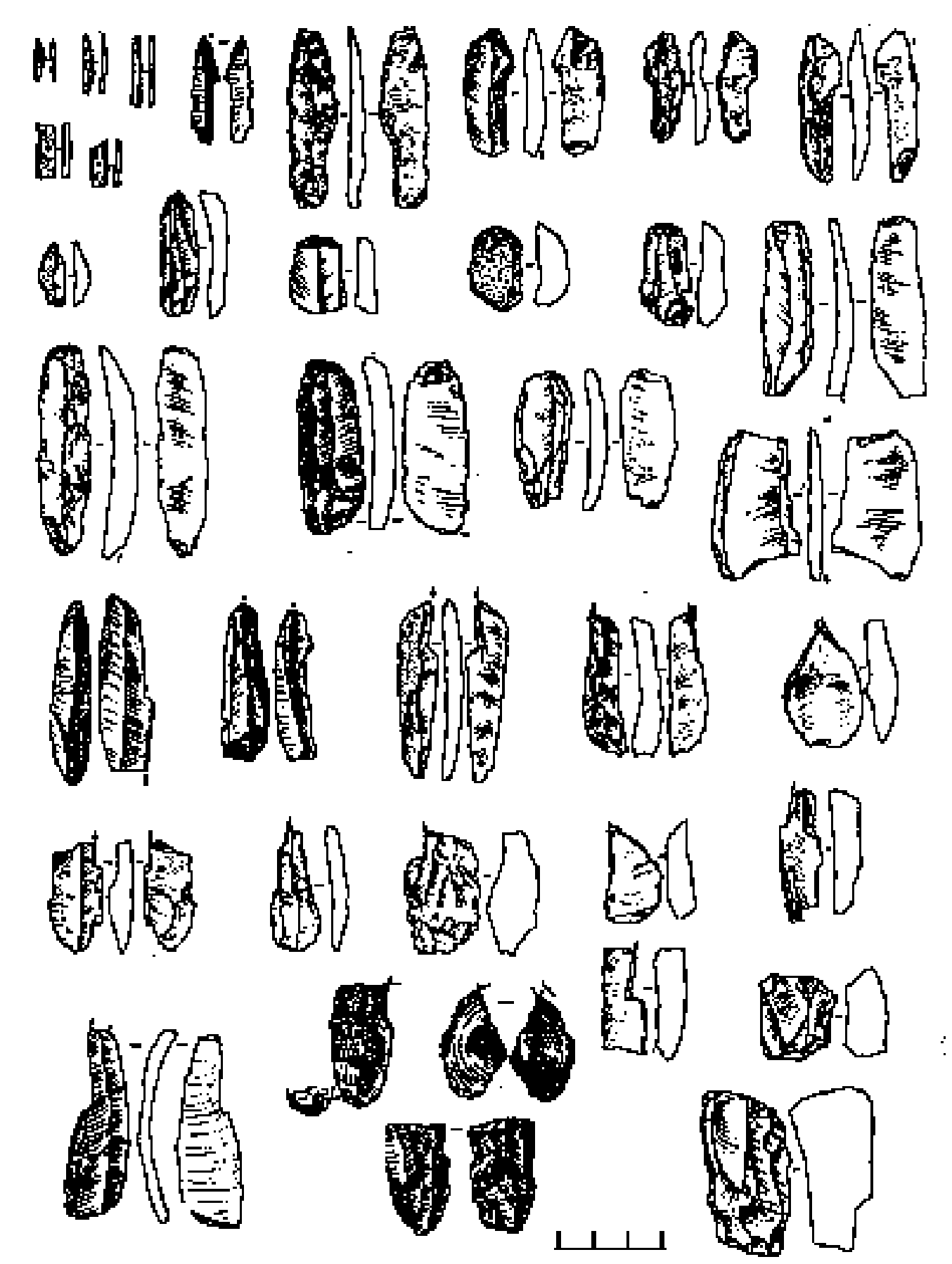

Figure 8 - Berdyj (d'après Kaletchitz 1984).

Figure 8 - Berdyj (After Kaletchitz 1984). 

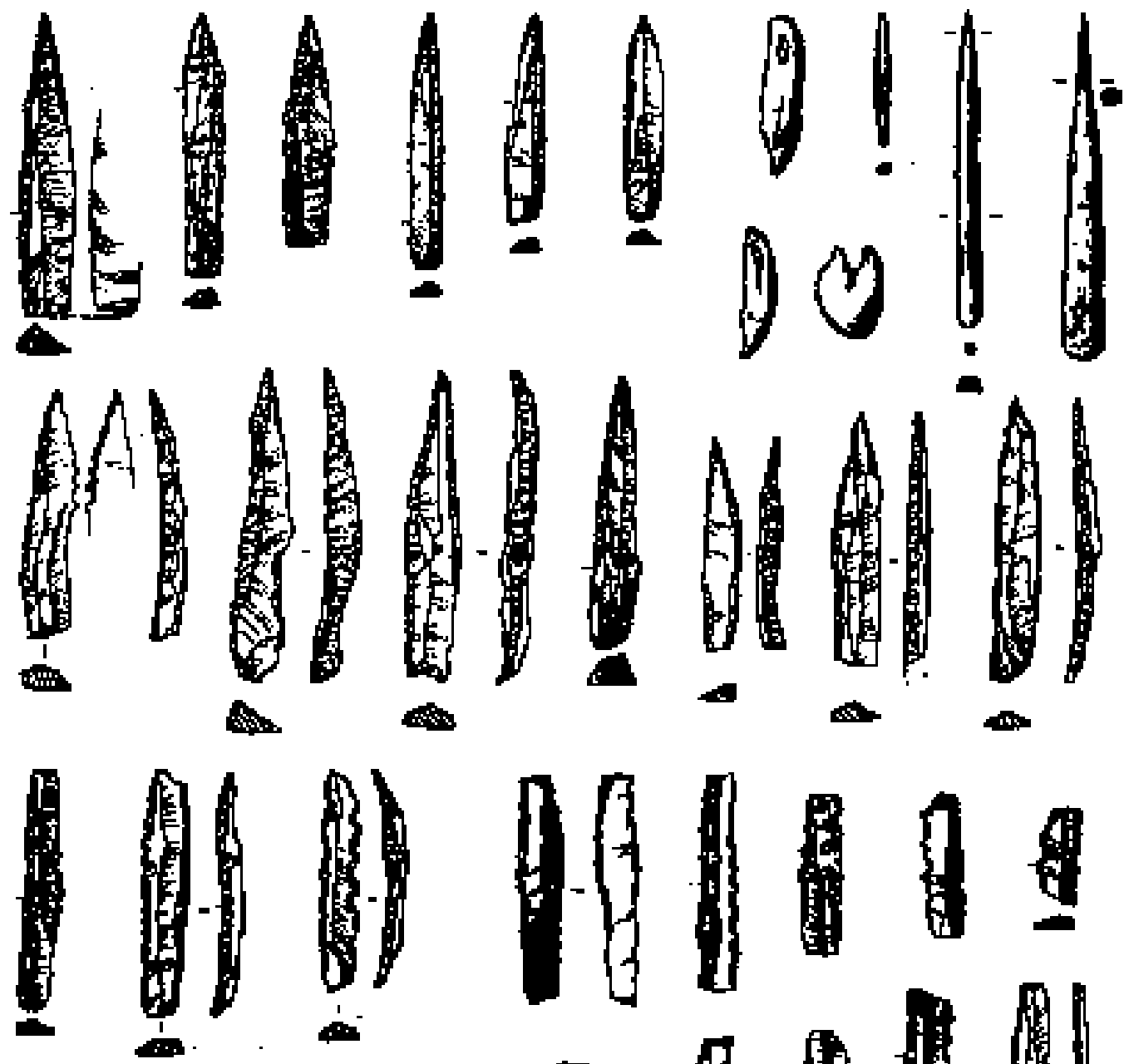

- 11 4\%

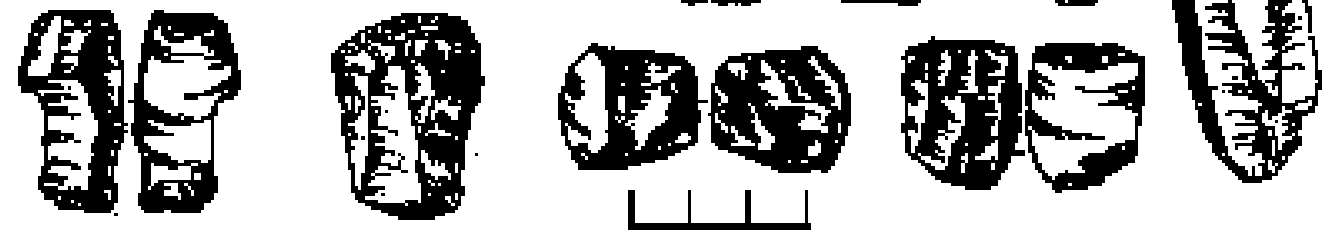

Figure 9 - Kostienki 4 (II) (d'après Rogatchev 1955).

Figure 9 - Kostienki 4 (II) (After Rogatchev 1955). 


\section{6-4.}
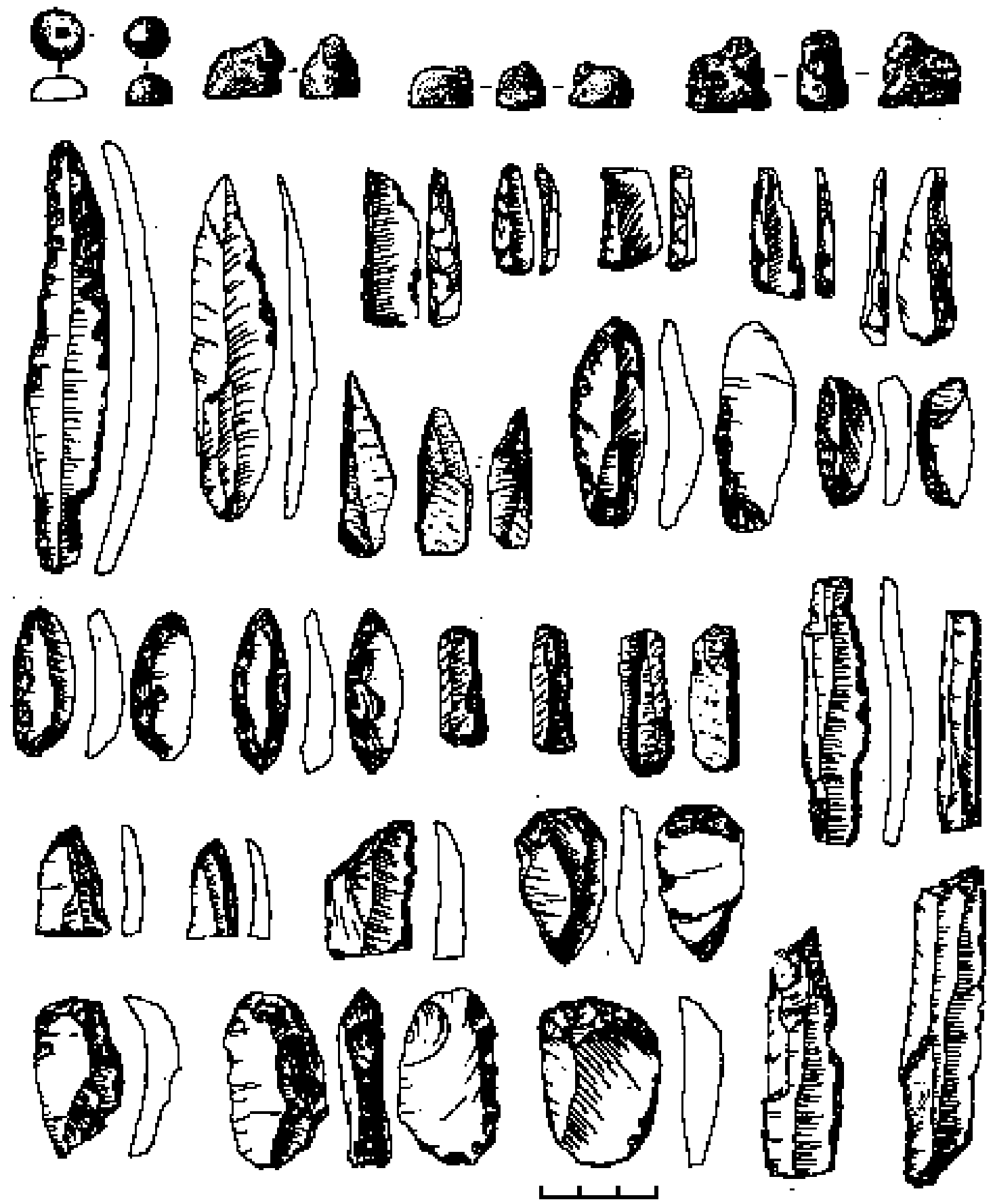

Figure 10 - Kostienki 11 (Anosovka 2) (II) (d'après Rogatchev 1961, 1962; Popov 1983; Popov et Poustovalov, 2004). Figure 10 - Kostienki 11 (Anosovka 2) (II) (After Rogatchev 1961, 1962; Popov 1983; Popov and Poustovalov 2004). 


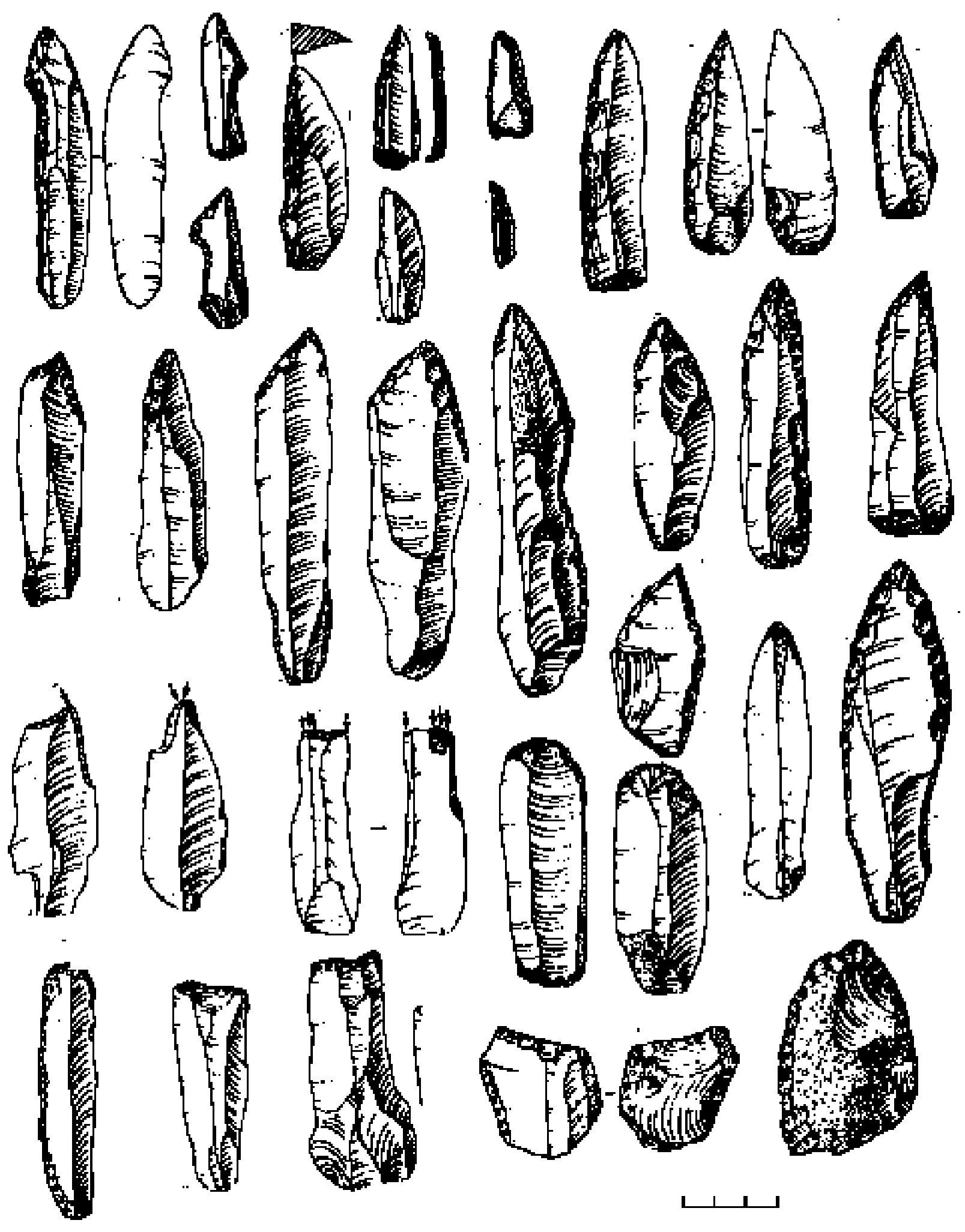

Figure 11 - Poushkari (d'après Boriskovski 1953, 1984).

Figure 11 - Poushkari (After Boriskovsky 1953, 1984). 

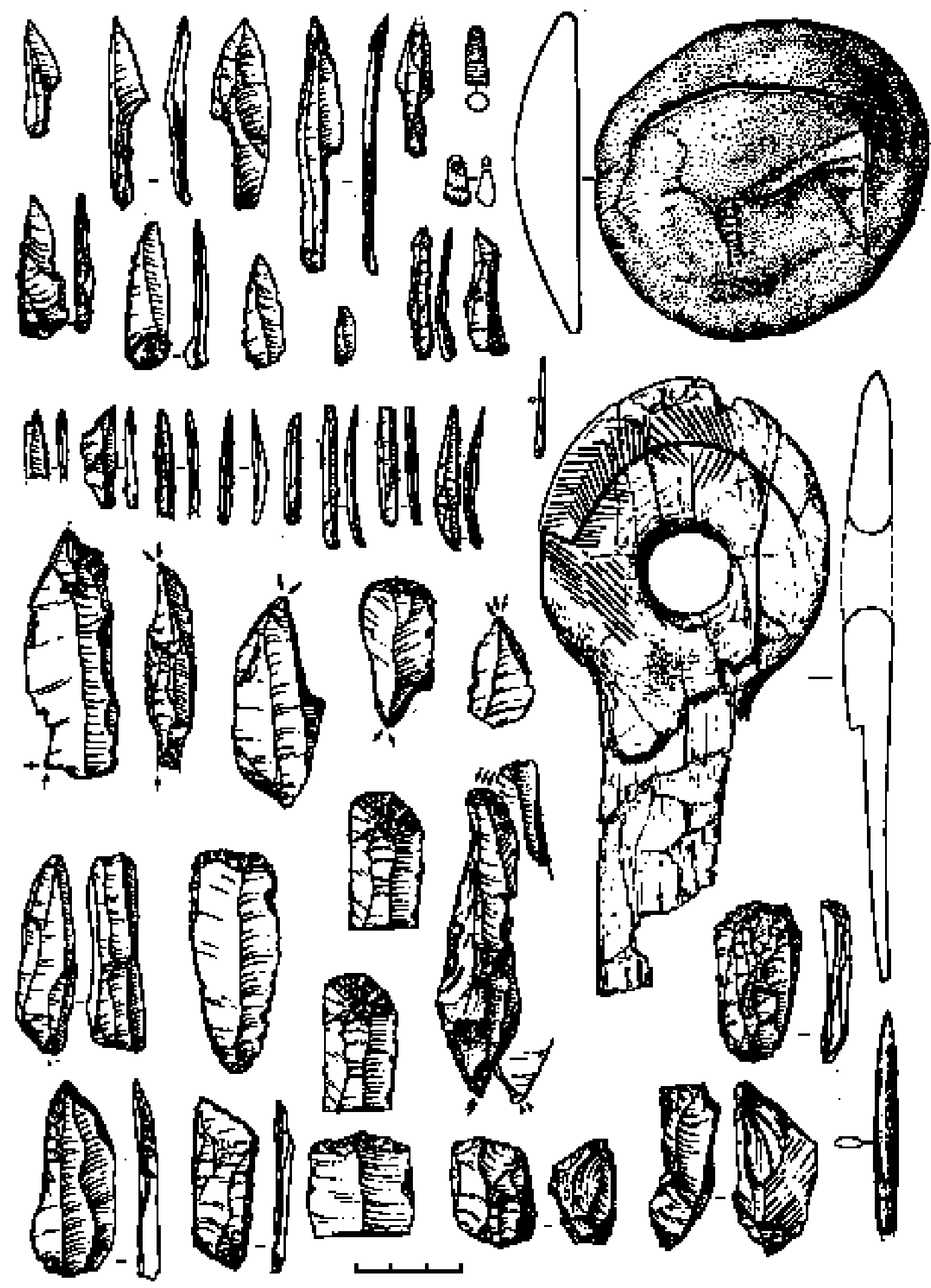


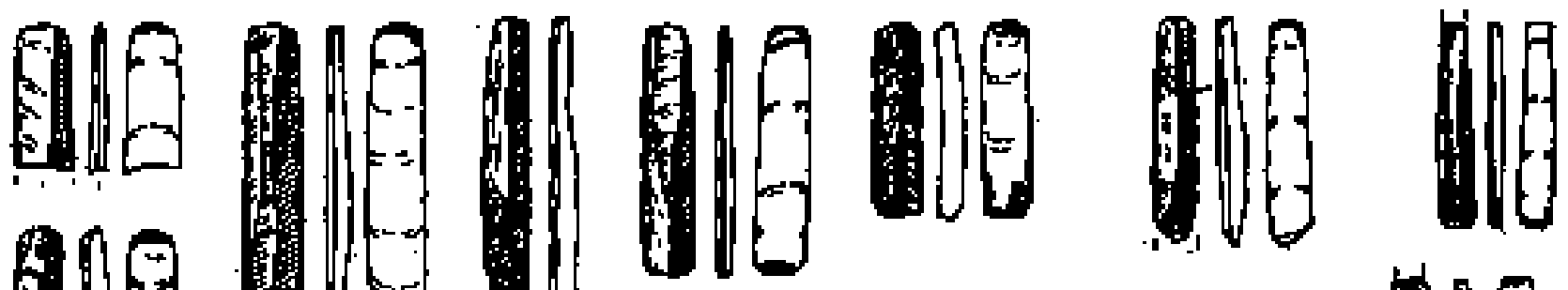

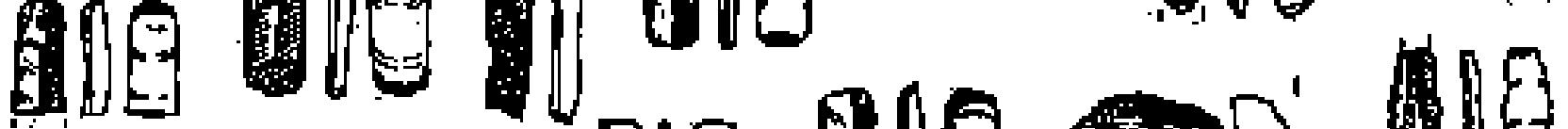

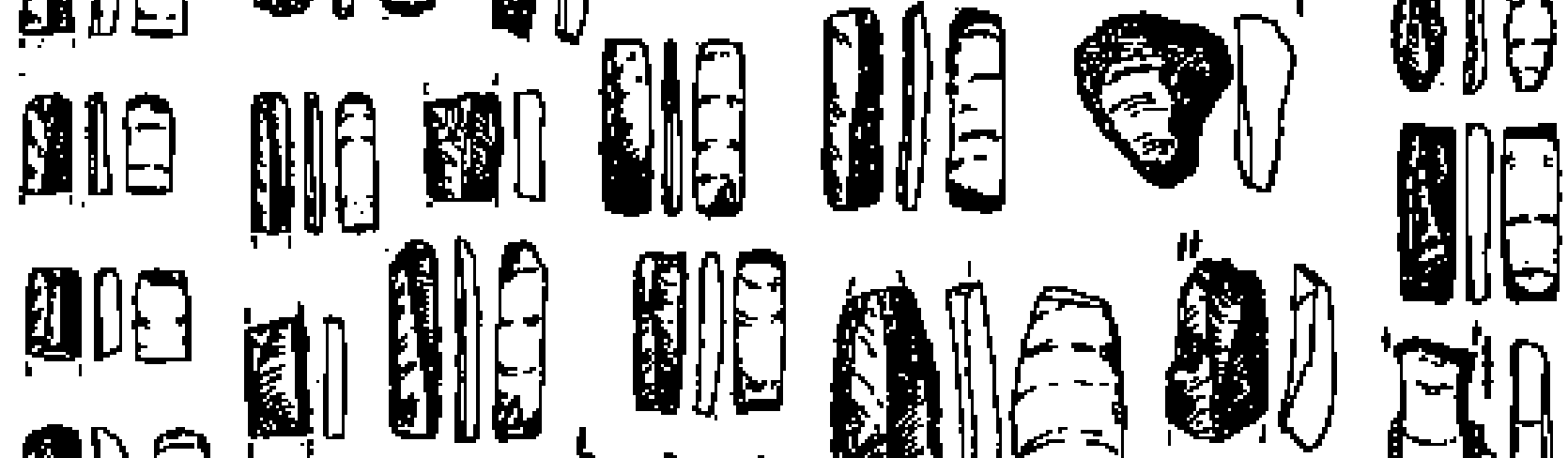

109

6)
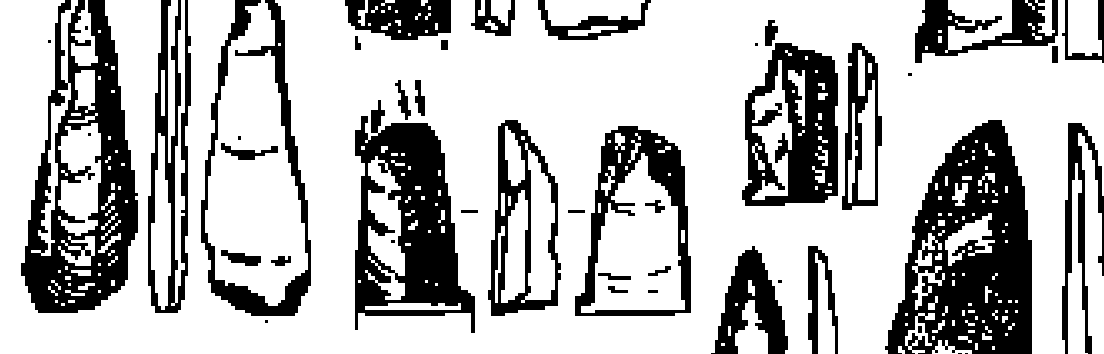

6 1 10
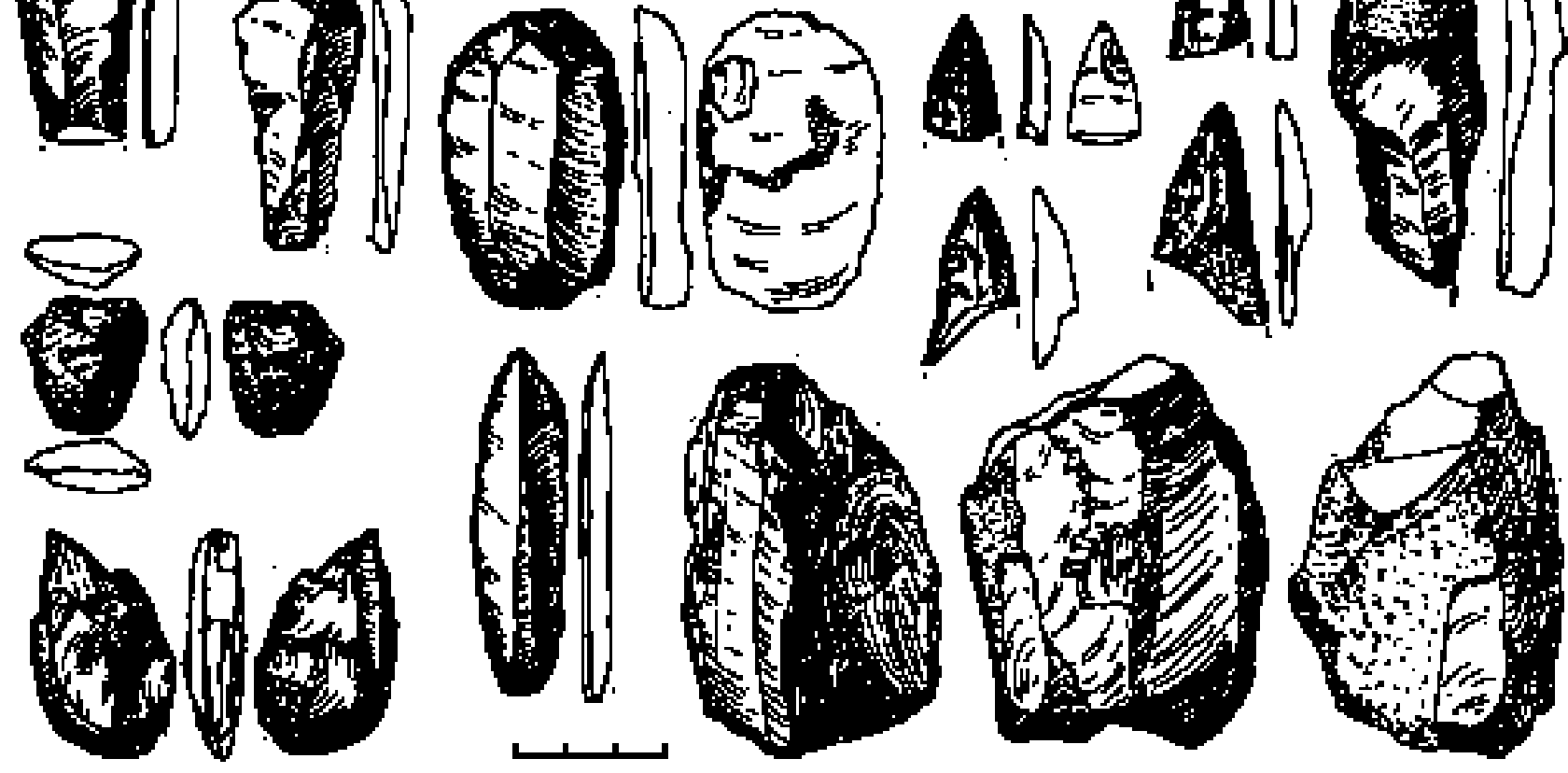

Figure 13 - Borschevo 5 (d'après Lissitsyn 2004).

Figure 13 - Borschevo 5 (After Lissitsyn 2004). 
- des pointes foliacées symétriques ayant un profil planoconvexe ou bi-convexe formé par une retouche bifaciale partielle ;

- des pointes et lames à bord abattu constituant près de $50 \%$ de l'outillage. Le caractère particulier de ce groupe est l'existence d'une série d'objets similaires aux federmesser ou aux pointes aziliennes, associés à des pointes diverses du Gravettien. Le problème de la distinction des pointes gravettiennes et des federmesser fut débattu dans les années 1970. II est d'importance puisque cette variété de pointes à dos est un des fossiles directeurs de l'industrie, de Kostienki 11 (II), mais aussi de assemblages de Kostienki 21(III), Poushkari 1 et Kliussy.

L'association «hybride» inhabituelle de types d'outils dans l'assemblage lithique de la couche II de Kostienki 11, n'a pas d'analogie avec le Paléolithique supérieur d'Europe de l'Est. Néanmoins, quelques similitudes ponctuelles pourraient avoir une certaine importance.

Les figurines animales de Kostienki 11 (II) sont presque identiques aux figurines de Kostienki 4 (I) et dans une moindre mesure de Kostienki 1 (I) (Rogatchev, 1962; Abramova, 1961; 1967; Anikovich, 1983). Les pointes à dos du «groupe de federmesser» de Kostienki 11 (II) sont bien connues dans les industries des sites Poushkari 1 et Kliussy dans le bassin du Dniepr et dans l'industrie de Kostienki 21 (III). L'interprétation de ce phénomène est délicate comme pour toutes analogies partielles. Dans tous les cas, cela n'est pas accidentel puisque des éléments culturels très importants sont concernés.

3.3.1 - Les industries associées à la culture du type de Kostienki 11 (II)

Les deux assemblages de Poushkari et Klussy sont des industries similaires à bien des égards, au même titre que l'ensemble Kostienki 1 (I) - Avdeevo.

Le site de Poushkari 1(fig. 11) est caractérisé par la construction d'habitat en os de mammouth avec une ligne de trois foyers. L'assemblage lithique comporte des pointes diverses dont certaines foliacées unifaciales, et des pointes à dos y compris celles de type fedemesser. Les indices de burins et de grattoirs sont bas. Cette composition révèle un type culturel original, distinct des industries contemporaines de Kostienki et des sites voisins du bassin de Dniepr (Roudinski, 1947; Boriskovski 1953 et 1984 ; Beliaeva 1997a et b ; 2000, 2002 et 2004). Les âges de $21100 \pm 400$ (GIN - 3381) et $22350 \pm 150$ (GIN - 3381) sont généralement admis pour cette industrie.

Bien que nous ne disposions que d'informations préliminaires sur le site et sur l'industrie lithique de Klussy (Shovkoplias 1967 ; Nuzhnij 1992) sa similitude avec Poushkari 1, ne fait pas de doute.

\section{4 - Variété du type de Kostienki 21 (niveau III)}

Malgré de fortes ressemblances, l'assemblage de la couche culturelle III de Kostienki 21, met en évidence l'existence d'une autre unité culturelle originale du
Gravettien de Kostienki. Les traits spécifiques concernent tous les éléments de la culture matérielle : l'assemblage lithique et osseux, l'art, la parure (Praslov et Rogachev 1982 ; Praslov 1985 ; Ivanova 1981 et 1985). Pour l'industrie lithique, en premier lieu, les proportions de pointes à cran sont celles qui ont été observées dans le Gravettien de l'Ouest, de même que l'importance des pointes à cran du Gravettien oriental et les pointes à dos du type federmesser. L'industrie osseuse est caractérisée par des types particuliers d'outils dont la fonction est inconnue. L'art mobilier est représenté par des gravures d'animaux sur plaquettes de pierre, unique pour l'Europe de l'Est, mais bien connues à l'Ouest (fig. 12). Les datations obtenues sont ; $22270 \pm 150$ (GrN - 7363), $21260 \pm 340$ (GrN - 10513).

\section{5 - Variété du type de Kostienki 9 - Borschevo 5}

Ce faciès de Gravettien de la règion de KostienkiBorschevo se distingue par de fortes proportions de lamelles à dos abattu tronquées parfois aux deux extrémités (rectangles), formées par une retouche directe ou bidirectionnelle. La ressemblance entre les assemblages de Kostienki 9 (Biriutchii log) et Borschevo 5 (I) est également soulignée par des pointes symétrique ou asymétrique dont les bords portent une retouche semi-abrupte parfois importante (Litovtchenko 1966b ; Praslov et Rogachev 1982 ; Lissitsyn 2004) (fig. 13)

\section{4 - CONCLUSIONS}

1- Les données actuelles, et en premier lieu les datations absolues, mettent en évidence une apparition polycentrique du Gravettien. Les données de l'Abri Pataud en Aquitaine, Paglicci en Italie, Masière-Canal en Belgique, Pavlov I, Dolni Vestonice I, Brillenhöhle en Europe centrale et de nombreux autres sites. (Djindjian et al. 1999) soulignent que le caractère indépendant et simultané de l'émergence de technocomplexes gravettiens dans les niveaux datés de 28-29 ka à Kostienki 8 (II) est un des éléments du processus pan-européen de l'apparition spontanée du technocomplexe gravettien. C'est actuellement, sa manifestation la plus orientale ${ }^{3}$.

2 - L'apparition du Gravettien a eu lieu probablement au cours de l'oscillation tempérée d'Arcy-Denecamp (second groupe chronologique de Kostienki). Elle résulte de mutations survenues au sein des techno-complexes, indépendamment de la mise en place de nouvelles conditions climatiques. La période 28-29 ka est une période tempérée et stable sans fluctuations climatiques.

3 - Au cours de 28-29 ka, la structure bimodale du Paléolithique supérieur ancien (l'Aurignacien pan-européen et les cultures locales de «transition») est remplacée par une structure unimodale correspondant aux technocomplexes gravettiens unifiés de structure «mosaïque» qui marque le début du Paléolithique supérieur moyen.

4 - La technologie aurignacienne basée sur une production de lames étroites et de microlamelles à partir de nucléus uni- 
polaires a été remplacée par la technologie mettant en œuvre des nucléus bipolaires produisant trois types de supports : lame, lamelle et microlamelle. Les diagnoses culturelles fondées sur les formes spécifiques de l'activité domestique, ont été remplacées par la prise en compte des formes spécifiques des projectiles ou des armatures de jet traditionnellement liés à la chasse.

5 - Les particularités évidentes du Gravettien de Kostienki s'inscrivent dans un processus qui a conduit, en Europe, à la différenciation de faciès locaux à partir d'un technocomplexe global. II est important de noter que des ressemblances se manifestent avec le Gravettien européen occidental au sein du groupe Gravettien évolué [Kostienki 4 (II) et Kostienki 21 (III)] et qu'elles coexistent avec des variantes propres à l'Europe de l'Est comme les faciès de KostienkiAvdeevo et de Kostienki 11 (II).

\section{Remerciements}

Je remercie très chaleureusement Monsieur J.-Ph. Rigaud pour m'avoir invité à participer à la table ronde «Entités régionales d'une paléoculture Européenne : le Gravettien» et pour la correction de mon texte français.

\section{BIBLOGRAPHIE}

ABRAMOVA Z.A. 1961 - Les images animales du site paléolithique Alexandovka. - Les Communications brèves d'Institut d'Archéologie, vol. 82. Moscou, p. 97-103 (en russe).

ABRAMOVA Z.A. 1962 - L'art paléolithique sur le territoire de I'URSS. Le corps des Sources Archéologiques, vol. A4-3. Moscou-Leningrad (en russe).

ABRAMOVA Z.A. 1966 - L'image de l'Homme dans l'art paléolithique d'Eurasie. Moscou-Leningrad (en russe).

ABRAMOVA Z.A. 1967 - L'art mobilier paléolithique en URSS. - Quartär, Bd. 18, p. 99-126.

ABRAMOVA Z.A. 2005 - Les animaux et l'Homme dans l'art paléolithique d'Europe. St.-Petérsbourg (en russe).

AMIRKHANOV Kh.A. 1998 - Gravettien oriental ou les industries gravettoïdes d'Europe Centrale et Orientale. In : Gravettien oriental /ed. Kh.A.Amirkhanov/. Moscou, p.1534 (en russe).

AMIRKHANOV Kh.A. 2000 - Le site de Zaraisk. Moscou (en russe).

AMIRKHANOV Kh.A., LEV S.Yu. 2004 - La statuette de Bison du site de Zaraisk. IN: Les problèmes de l'Age de Pierre de la Plaine Russe /ed. Kh.A. Amirkhanov/. Moscou, p. 299-321 (en russe).

AMIRKHANOV Kh.A., LEV S.Yu. 2007 - New finds of Paleolithic art from Zaraisk. in : Rossijskaia Archeologia 1. Moscou p. 22-35 (en russe).
ANIKOVITCH M.V. 1983 - À propos du problème de la synchronisation de certains sites paléolithiques de la région Kostienki-Borschevo. - Les Communications brèves de I'Institut d'Archéologie de l'Académie des Sciences d'URSS, vol. 173. Moscou, p.16-23 (en russe).

ANIKOVITCH M.V. 1998 - Le domaine de Dniepr-Don culturel-historique des chasseurs de mammouths : du «Gravettien oriental» à «l'Epigavettien oriental». In : Gravettien oriental /ed. Kh.A.Amirkhanov/. Moscou, p. 3566 (en russe).

BELIAEVA V.I. 1977 - Essai de création d'une méthode pour la description des "couteaux du type de Kostienki». IN: Problèmes du Paléolithique d'Europe Orientale et Centrale / ed. N.D.Praslov /. Leningrad, p. 117-126 (en russe).

BELIAEVA V.I. 1979 - L'assemblage lithique de Kostienki I. (L'essai de la classification). - Résumé de la thèse de doctorat. Leningrad (en russe).

BELIAEVA V.I. 1997a - Les éléments gravettiens de l'industrie de Poushkari 1. In : «La Gravettien Oriental» (résumé des communications pour le colloque intemational. Zaraisk-Moscou). Moscou, p. 13-16 (en russe).

BELIAEVA V.I. 1997b - Les excavations du terrain nouveau sur le site paléolithique Poushkari I. In : Le recueil de Poushkari. Vol.I. St.-Petérsbourg, p. 5-18 (en russe).

BELIAEVA V.I. 2000 - Poushkari I. La caractéristique du site. - L'Almanach archéologique, vol. 9. Donetsk, p. 145152 (en russe).

BELIAEVA V.I. 2002 - L'industrie lithique de Poushkari I. IN: Paléolithique supérieur - Pléistocène supérieur : les dynamiques des événements naturels et de la périodicité des cultures archéologiques / Éd. N.D. Praslov / (Les matériaux du colloque International, consacré au 90 e anniversaire de A.N. Rogatchev). St.-Petérsbourg, p. 133-137(en russe).

BELIAEVA V.I. 2004 - Tools and morphological forms flint's industry of Pushkari sites. - L'Almanach archéologique, vol. 16. Donetsk, p. 251-262 (en russe).

BESSOUDNOV A.A. 2005 - Les variétés gravettiennes dans la région paléolithique de Kostienki-Borschevo. IN: The young archaeologists almanac. 2005 /ed. V.E. Eremenko/. St.-Petersburg, p. 11-21(en russe).

BORDES F. 1968 - Emplacements de tentes du Périgordien supérieur évolué. Quartär, t. 19.

BORISKOVSKY P.I. 1953 - Paléolithique d'Ukraine. Essais historico-archéologique. In : Matériaux et recherches sur l'Archéologie de l'URSS, vol. 40. Moscou-Léningrad (en russe).

BOROSKOVSKY P.I. /ed./ 1984 - Paléolithique de I'URSS. Série : Archéologie de l'URSS. Moscou-Leningrad (en russe). 
BOUDKO V.D. 1964 - À propos des structures d'habitats du site paléolithique de Berdyje. - Les Communications brËves de l'Institut d'Archéologie de l'Académie des Sciences d'URSS, vol. 101. Moscou, p.31-34 (en russe).

BOUDKO V.D. 1970 - Le Paléolithque. In: Essais sur l'archéologie de Biélorussie, pt. 1. Minsk (en russe).

BOUDKO V.D., SOROKINA S.A. 1969 - Le Paléolithique supérieur du nord-ouest de la Plaine Russe. IN: Milieu et développement de la sociéte préhistorique dans la partie Européene de I'URSS /ed. I.P. Guerassimov/ (pour le VIII Congrés de I'INQUA, Paris, 1969). Moscou, p. 127-136 (en russe).

DJINDJIAN F., KOZLOWSKI J., OTTE M. 1999 - Le Paléolithique supérieur en Europe. Paris.

EFIMENKO P.P. 1928 - Certains bilans d'étude du Paléolithique d'URSS. L'Homme, 1, Moscou, p. 45-59 (en russe).

EFIMENKO P.P. 1953 - La société préhistorique. Kiev (en russe).

EFIMENKO P.P. 1956 - À propos de la question sur le caractère du processus historique du Paléolithque supérieur d'Europe de l'Est (sur les sites des types szeletien et grimaldien soi-disant). - L'Archéologie soviétique, t. XXVI, p. 28-53 (en russe).

EFIMENKO P.P. 1958 - Kostienki 1. Moscou-Léningrad (en russe).

EFIMENKO P.P. 1960 - Les éléments du Proche Orient dans les assemblages du Paléolithque supérieur du Nord de la mer Noire (sur l'origine de Magdalénien d'Europe de l'Est). - L'Archéologie soviétique, 4, p. 14-25 (en russe).

GAVRILOV K.N. 2002 - On the cultural attribution of the Upper Paleolithic settlement Khotylevo. IN: The Upper Paleolithic - Upper Pleistocene: dynamics of natural events and periodization of archaeological cultures (abstracts of the international conference, dedicated to 90-th birth anniversary of A.N. Rogachev) /ed. N.D.Praslov/. StPetersburg, p. 143-147 (en russe).

GAVRILOV K.N. 2004 - La typologie de l'indusrie lithique et l'attribution culturelle du site de paléolithique supérieur de Khotylevo. IN: Les problèmes de l'âge de pierre de la Plaine Russe /ed. Kh.A. Amirkhanov/. Moscou, p. 262-284 (en russe).

GIRIA Y., BRADLEY B. 1998 - Blade technology at Kostenki 1/1, Avdeevo and Zaraisk. IN: Gravettien oriental /ed. Kh.A. Amirkhanov/. Moscou, p. 191-213.

GRIGOREV G.P. 1993 - The Kostenki-Avdeevo archaeological culture and the Willendorf-Pavlov-Kostenki-Avdeevo cultural unity. IN: From Kostenki to Clovis. Upper Paleolithic - Paleo-Indian Adaptations /eds. O. Soffer, N.D. Praslov/. New-York, p. 51-65.

GVOZDOVER M.D. 1961 - Les traits particuliers de l'assemblage lithique du site paléolithique de Avdeevo - Les
Communications brèves de l'Institut d'Archéologie de l'Académie des Sciences d'URSS, vol. 82. Moscou, p. 112-119 (en russe).

GVOZDOVER M.D. 1995 - Art of Mammoth Hunters. The finds from Avdeevo. Oxford Monograph, 49.

GVOZDOVER M.D. 1998 - L'assemblage lithique du site du Paléolithique supérieur Avdeevo. IN: Gravettien oriental /ed. Kh.A. Amirkhanov/. Moscou, p. 224-278 (en russe).

IVANOVA M.A. 1981 - Complexe d'habitat du site paléolithique de Gmelin en Kostienki. - Les Communications brèves de l'Institut d'Archéologie de l'Académie des Sciences d'URSS, vol. 165. Moscou, p. 37-42 (en russe).

IVANOVA M.A. 1985 - La structure d'habitat paléolithique du site de Gmelin. - Le résumé de la thèse de doctorat. St.Pétersbourg (en russe).

KALETCHETS E.G. 1984 - Le peuplement initial du territoire de Biélorussie. Minsk (en russe).

KOZLOWSKI J.K. 1969 - Problem tzw. kultury Kostienkowsko-Willendorskiej. Cz.l. Analiza inwentarzy kamiennych. - A rcheologia Polski, t. XIV, zesz. 1, p. 19-85.

KOZLOWSKI J.K. 1970 - Problem tzw. kultury Kostienkowsko-Willendorßkiej. Cz.II. Analiza inwentarzy koscianych I zabytków sztuki. - Prace Archeologiczne, zesz. 12, Kraków, s. 27-46.

KOZLOWSKI J.K. 1986 - The gravettian in Central and Eastem Europe. - Advances in World Archaeology, vol. 5, p.131-200.

KOZLOWSKI J.K. 1998 - La géochronologie de l'horizon à pointes à cran en Europe Centrale. IN: Gravettien oriental /ed. Kh.A. Amirkhanov/. Moscou, p. 81-89.

KSENZOV V.P. 1988 - Paléolithique et Mésolithique du bassin de Dniepr de Biélorussie. Minsk (en russe).

LEV S.Yu., AMIRKHANOV Kh.A. 2002 - Comparative description and stylistic analysis of the bison statuette from Zaraiskaja site. - Archaeology, ethnography and anthropology of Eurasia, $n^{\circ} 3$ (11). Novosibirsk. p. 22-31 (en russe).

LISSITSYN S.N. 2004 - Chronostratigraphy of the site Borshchevo 5 on the evidences of 2002-2003 seasons of excavations. IN: Kostenki \& the Early Upper Paleolithic of Eurasia: general trends, local developments. Guidebook and abstracts of the Field seminar dedicated to the 125-th anniversary of the discovery of the Paleolithic in Kostenki /eds. M.V. Anikovich, N.I. Platonova/. Voronezh, p. 66-79 (en russe).

LITOVTCHENKO L.M. 1966a - À propos du groupe des constructions d'habitat de la couche culturelle 2 du site Telmanskaia. IN: Les problèmes d'histoire et d'archéologie leds. G.S. Meliankov, Yu.I. Afonin/. Minsk, p. 298-305 (en russe). 
LITOVTCHENKO L.M. 1966b - Le site paléolithique de Biriutchiy Log (Kostienki IX). - Les nouvelles de l'Académie des Sciences de BSSR. Série des sciences sociales, $n^{\circ} 3$ (en biélorusse).

LITOVTCHENKO L.M. 1969 - Les nouveaux matériaux sur la station paléolithique Telmanskaia. - L'Archélogie sovietique, 3. Moscou, p. 110-123 (en russe).

NUZHNIJ D.Yu. 1992 - L'évolution de la technique microlithique dans l'âge de pierre. Kiev (en ukrainien).

OTTE M., NOIRET P., CHIRICA V., BORZIAK I. 1996 - Rythme évolutif du Gravettien Oriental. IN: XIII Congrès International d'U.I.S.P.P., Section 6: The Upper Palaeolithic /eds. A. Palma di Cesnola, A. Montet-White, K.Valoch/, Colloquim XII. The Origine of the Gravettian. Forli, p. 213-226.

PALMA DI CESNOLA A. 1996 - Le Gravettien le plus ancien en Italie. IN: XIII Congrès International d'U.I.S.P.P., Section 6: The Upper Palaeolithic /eds. A. Palma di Cesnola, A. Montet-White, K.Valoch/, Colloquim XII. The Origine of the Gravettian. Forli, p. 227-235.

POLIKARPOVITCH K.M. 1968 - Paléolithique du bassin de Dniepr supérieur. Minsk (en russe).

POPOV V.V. 1983 - L'analyse de l'industrie lithique du site Kostienki 11 (couche culturelle II). IN: Les sites préhistoriques sur le territoire d'Europe de l'Est /eds. A.T. Sinuk, A.L. Priakhine, I.B. Vassiviev, V.I. Pogorelov/. - Les nouvelles de l'Institut pédagogique de Voronej, t. 227. Voronej, p. 5-13 (en russe).

POPOV V.V., ANIKOVICH M.V., HOFFECKER J.F., DUDIN A.V., PUSTOVALOV A.Yu., CHERNYSHEV S.S. 2004 Kostenki (Anosovka 2). In: Kostenki \& the Early Upper Paleolithic of Eurasia: general trends, local developments. Guidebook and abstracts of the Field seminar dedicated to the $125^{\text {th }}$ anniversary of the discovery of the Paleolithic in Kostenki /eds. M.V. Anikovich, N.I. Platonova/. Voronezh, p. 6-17 (en russe).

POPOV V.V., PUSTOVALOV A.Yu. 2004 - L'habitat de la couche culturelle II du site Kostienki 11 (Anosovka 2). IN: Les sites archéologiques du bassin de Don /ed. A.T. Sinuk/, Voronej, p. 3-8 (en russe).

PRASLOV N.D. 1985 - Engraved animals in Gmelin Paleolithic site (Kostenki 21). IN: Mammals of Northem Eurasia during Quatemary /eds. N.K. Vereshchagin, I.E. Kuzmina/. - Travaux de l'Institut Zoologique AS URSS, t. 131. Leningrad, p. 114-120 (en russe).

PRASLOV N.D., ROGACHEV A.N. /eds./. 1982 Palaeolithic of the Kostenki-Borshchevo area on the river Don. 1879-1979. Results of field investigations. Leningrad (en russe).

PRASLOV N.D., SOULERJYTSKY L.D. 1997 - De nouvelles données chronologiques pour le Paléolithique de
Kostienki-sur-Don. - PréhistoireEuropéenne, vol.11, Liège, p. 133-143.

ROGATCHEV A.N. 1955 - L'habitat d'Alexandrovskoe de l'âge de pierre ancien en Kostienki sur Don. - Matériaux et recherches sur l'Archéologie de I'URSS, vol.45. MoscouLeningrad (en russe).

ROGATCHEV A.N. 1957 - Les sites à plusieurs couches culturelles de la région de Kostenki-Borschevo sur Don et le problème de l'évolution de la culture du Paléolithique supérieur dans la Plaine Russe. - Matériaux et recherches sur l'Archéologie de l'URSS, vol. 59. Moscou-Leningrad, p. 59-134 (en russe).

ROGATCHEV A.N. 1961 - Anosovka II - Le site nouveau à plusieurs couches culturelles de Kostienki. - Les Communications brèves de l'Institut d'Archéologie de l'Académie des Sciences de l'URSS, vol. 82. Moscou, p. 8696 (en russe).

ROGATCHEV A.N. 1962 - Les sculptures schématiques des animaux de Kostienki (Anosovka II). - IN: Abramova Z.A. 1962. L'art paléolithique sur le teritoire de I'URSS. Le corps des Sources Archéologiques, vol. A4-3. MoscouLeningrad p. 78-80 (en russe).

ROUDINSKY M.la. 1947 - Poushkari. - L'Archéologie soviétique, IX. Moscou-Leningrad, p. 171-198 (en russe, res.fr).

SELEZNEV A.B. 1998 - La technologie du débitage lithique du site de Khotylevo 2. In: Gravettien oriental / ed. Kh.A.Amirkhanov/. Moscou, p. 214-225 (en russe).

SHOVKOPLIAS I.G. 1967 - L'expédition pour la recherche du Paléolithique en 1965-1966. IN: Les recherches archéologiques en Ukraine dans les années 1965-1966, vol. 1/ed.P.P. Tolotchko/. Kiev, p. 56-61 (en russe).

SINITSYN A.A. 1999 - Chronological problems of the Palaeolithic of Kostenki-Borschevo area: geological, palynological and ${ }^{14} \mathrm{C}$ perspectives. $/ /{ }^{14} \mathrm{C}$ et Archéologie. $3{ }^{\text {ème }}$ Congrès International (Lyon, 6-10 avril 1998) /eds. J.Evin, Ch.Oberlin, J.-P.Daugas, J.-F.Salles/. Mémoires de la Société Préhistorique Francaise, t.XXVI et Supplément 1999 de la Revue d'Archéomètrie. Lyon, p. 143-150.

SINITSYN A.A. 2003a - A Palaeolithic 'Pompeii' at Kostenki, Russia. - Antiquity, vol. 77, n. 295, p. 9-14.

SINITSYN A.A. 2003b - The most ancient sites of Kostenki in the context of the Initial Upper Paleolithic of northern Eurasia. IN: The Chronology of the Aurignacian and of the Transitional Technocomplexes. Dating, Stratigraphies, Cultural Implications (Proccedings of Symposium 6.1 of the $\mathrm{XIV}^{\text {th }}$ Congress of the UISPP) /eds. J. Zilhao, F. d'Errico/ Trabalhos de Arqueologia, 33. Lisboa, p. 89-107.

SINITSYN A.A., PRASLOV N. D., SVEZHENTSEV Yu. S., SULERZHITSKIY L. D. 1997 - Radiocarbon chronology of the Upper Paleolithic of Eastern Europe. IN: Radiocarbon 
Chronology of the Paleolithic of Eastern Europe and Northem Asia. Problems and perspectives /eds. A.A. Sinitsyn, N.D. Praslov/. St. Petersburg, p. 21-66 (en russe, résumés en anglais).

SOFFER O. 1987 - Upper Paleolithic connubia, refugia, and the archaeological record from Eastern Europe. IN: The Pleistocene Old World. Regional Perspectives / ed. O. Soffer/. New York, London, p. 333-349.

SOFFER O. 1993a - Migration vs. Interaction in Upper Palaeolithic Europe. IN: Cultural Transfomations and Interpretations in Eastern Europe /eds. J. Chapman, P. Dolukhanov/. - Worldwide Archaeological Series, vol.6. Newcastle-upon-Tyne, p. 65-70.

SOFFER O. 1993b - Upper Paleolithic adaptations in Central and Eastern Europe and Man-Mammoth interactions. IN: From Kostenki to Clovis. Upper Paleolithic Paleo-Indian Adaptations /eds. O. Soffer, N.D. Praslov/. New-York, p. 31-49.

TARASSOV L.M. 1979 - Le site de Gagarino et sa place dans le Paléolithique européen. Leningrad (en russe).

TCHELIDZE L.M. (LITOVTCHENKO) 1968 - Le site de Telmanskaya et certaines questions de l'évolution de la culture paléolithique d'Europe de l'est. Thèse de doctorat. Leningrad (Archive d'IHMC RAS, fonds 35, l'inventaire 2, n. 193, 194) (en russe).

TROUSSOV A.V. 1994 - La couche culturelle du site du Paléolithique supérieur de Zaraisk. IN: Antiquités du bassin d'Oka /ed. G.F. Poliakova /. - Travaux du Musée Historique d'Etat, vol. 85. Moscou, p. 94-116 (en russe).
TROUSSOV A.V. 1998 - L'assemblage lithique du site paléolithique de Zaraisk. IN: Gravettien oriental /ed. Kh.A.Amirkhanov/. Moscou, p. 279-298 (en russe).

TROUSSOV A.V. 2002 - La couche culturelle du site paléolithique de Zaraisk (la morphologie - l'aspect stratigraphique). IN: Trends in the evolution of the East European palaeolithic /eds. A.A. Sintsyn, V.Ya. Sergin, J.F. Hoffecker/. Kostenki in the context of the Palaeolithic of Eurasia. Proccedings of Kostenki expedition IHMC RAS, ser. Research. vol.1. St.-Petersburg, p. 237-246 (en russe)

ZAMIATNINE S.N. 1934 - La station aurignacienne de Gagarino et les données nouvelles qu'elle fournit sur les rites magiques des chasseurs quaternaries. - Bulletin de l'Académie d'Histoire de la Culture Matérielle, fasc. 88. Moscou-Leningrad.

ZAVERNIAEV F.M. 1974 - Nouveau campement du Paléolithique supérieur sur la Desna. - L'Archéologie sovietique, 4. Moscou, p. 142-161 (en russe).

ZAVERNIAEV F.M. 1978 - La sculpture anthropomorphe du Paléolithique récent de la station de Khotylevo. L'Archéologie soviétique, 4. Moscou, p. 145-161(en russe).

ZAVERNIAEV F.M. 1991 - L'assemblage lithique du site du paléolithique supérieur de Khotylevo. - L'Archéologie soviétique, 4. Moscou, p.164-191(en russe).

ZAVERNIAEV F.M. 2000 - The remains of dwellings and hosehold assemblage at the Upper Palaeolithic site of Khotylevo. - Rossiyskaya Arkheologiya, 3, p. 69-87 (en russe). 
